\title{
Simulation of the flame describing function of a turbulent premixed flame using an open-source LES solver
}

\author{
Xingsi Han, Aimee S. Morgans* \\ Department of Aeronautics, Imperial College London, South Kensington campus, SW7 2AZ London, UK
}

\begin{abstract}
Numerical simulations were used to characterise the non-linear response of a turbulent premixed flame to acoustic velocity fluctuations. The test flame simulated was the bluff body stabilised flame which has been the subject of a detailed experimental study (Balachandran et al., 2005, Combustion E Flame). Simulations were performed using Large Eddy Simulation (LES) via the open source Computational Fluid Dynamics (CFD) software, Code_S aturne, with combustion modelled by combining a Flame Surface Density (FSD) method with a fractal approach for the wrinkling factor. The cold flow field and the unforced reacting flow were used for preliminary code validation. In order to characterise the non-linear response of the unsteady heat release rate to acoustic forcing, a harmonically varying velocity fluctuation, for which both the forcing frequency and normalised forcing amplitude were varied, was imposed. The flame response was characterised via a Flame Describing Function (FDF), also known as a nonlinear flame transfer function, for which the gain and phase shift depend on forcing amplitude as well as forcing frequency. The response at four frequencies was compared to experimental data in detail, confirming that the LES results captured both the qualitative flame dynamics and the quantitative response of the heat release rate with very reasonable accuracy. The full FDF was then obtained across more frequencies, again showing a good fit with the experimental data, other than for a slight under-prediction in gain, most probably due to neglecting the effect of wall heat loss and the effect of combustion modelling. The agreement was significantly better than has been obtained previously for this test case using numerical simulations. Finally, it was found that increasing combustor length had little affect on the flame response, which may prove useful for future long combustor stability and limit cycle analysis. This work thus confirms that LES, in this case via the open source Code_S aturne, provides a useful tool for characterising the response of lean premixed turbulent flames.
\end{abstract}

Keywords: Flame describing function (FDF), Combustion instability, Large eddy simulation, Premixed flame, Acoustic forcing, Code_Saturne

\section{Introduction}

Combustion in gas turbines, for example in power plants and aircraft jet engines, produces $\mathrm{NO}_{x}$ emissions that cause air pollution. Lean premixed combustion offers a means of reducing $\mathrm{NO}_{x}$ emissions, but at the expense of increased combustor susceptibility to damaging combustion instabilities [1]. These instabilities arise from the twoway coupling between the unsteady heat release rate from combustion and acoustic waves within the combustor. The two-way coupling forms a feedback cycle, which can lead to successively increasing amplitudes [2]. Combustion instability is noisy and the resulting vibration can severely damage the gas turbine. Therefore, the ability to predict and design-out instability during the design phase of a gas turbine combustor is crucial to achieving further reductions in $\mathrm{NO}_{x}$ emissions.

Accurately predicting the stability of a given combustor is the subject of a significant current research effort. Computational simulations must accurately incorporate unsteady combustion, acoustic waves, turbulence and heat

${ }^{*}$ Corresponding author. Tel.: +44 (0)20 75945053

Email addresses: x.han@imperial.ac.uk (Xingsi Han), a.morgans@imperial.ac.uk (Aimee S. Morgans) 
transfer, all over the (relatively) long timescales over which instabilities develop. This means that computational fluid dynamics (CFD) simulations of entire combustors, and the attached components which influence their behaviour, although possible, are impractical as an industry analysis tool. Low order combustor models are seen as a feasible alternative, e.g. $[3,4,5,6,7]$. Such models describe the combustor as a network of connected modules, and exploit the fact that one of the two phenomena key to the combustion instability mechanism, the acoustic waves, are very well modelled using simple analytical approaches. The response of the flame unsteady heat release rate to perturbations can be captured via a flame model $[8,9]$. The flame model should incorporate non-linearity at higher amplitudes, with this now established as being the main mechanism responsible for saturation into limit cycle in gas turbine combustors. In the past, very simple flame models were used $[3,4,5,6,7]$ for simulating general qualitative behaviour. However, more realistic flame models are now known to be needed to accurately predict stability boundaries and limit cycle amplitudes.

The flame model is traditionally defined via a (linear) flame transfer function (FTF) [10], given by,

$$
F(\omega)=\frac{Q^{\prime} / \bar{Q}}{u^{\prime} / \bar{u}}=G(\omega) e^{i \varphi(\omega)}
$$

where $Q^{\prime} / \bar{Q}$ is the normalised heat release rate fluctuation and $u^{\prime} / \bar{u}$ the normalised inlet velocity perturbation induced by the acoustic flow field. A linear model cannot predict the limit cycle amplitude [3, 11], nor other non-linear effects such as triggering and mode switching. Fairly recently, this concept has been extended to a non-linear flame transfer function $[4,5,6,7]$, also known as a Flame Describing Function (FDF) [12, 9, 13], defined as:

$$
F\left(\omega,\left|u^{\prime}\right|\right)=\frac{Q^{\prime} / \bar{Q}}{u^{\prime} / \bar{u}}=G\left(\omega,\left|u^{\prime}\right|\right) e^{i \varphi\left(\omega,\left|u^{\prime}\right|\right)}
$$

where the $\operatorname{FDF} F\left(\omega,\left|u^{\prime}\right|\right)$ is generally expressed in the frequency domain as gain (amplitude) $G\left(\omega,\left|u^{\prime}\right|\right)$ and phase $\varphi\left(\omega,\left|u^{\prime}\right|\right)$ which are functions of both forcing frequency $\omega$ and amplitude $\left|u^{\prime}\right|$. This approach makes the assumption of weak non-linearity. That is, the flame response to harmonic forcing is assumed to be primarily at the same frequency as the forcing, but with a gain and phase shift which depends upon the forcing amplitude as well as the frequency. By combining the FDF with a model for the combustor acoustics, the limit cycle amplitude can be determined.

Experimentally, several studies $[14,15,5,6,16,11,17,18,19,20]$ have been conducted to determine the flame models needed for stability analysis. Recent examples of this include Noiray et al. [9], who experimentally measured the FDF of an unconfined laminar burner; on combining with an acoustic model they obtained limit cycle predictions in excellent agreement with experiments. Palies et al. [21] extended this to a turbulent premixed swirling flame, while Silva et al. [22] combined a measured FDF for a premixed swirled combustor with a Helmholtz solver, predicting the limit cycle amplitude to within reasonable agreement with experimental data. Note that this approach has been used not only at lab scale, but also for real gas turbines operated at high pressure [20].

These experiments suggest that the gain of FDF tends to decrease as the amplitude of the applied velocity fluctuations increases, which can lead to saturation of heat release oscillations $[6,16,17,9,18,19]$. The physical cause of the saturation may be the interaction of the flame front with coherent structures $[6,16]$, attachment point dynamics [23] or flame quenching [18]. It has also been shown that the phase of the FDF may change with forcing amplitude, which may cause saturation due to a change in the nature of the Rayleigh source term driving combustion instability [9]. Swirl number fluctuations $[24,25]$ and the phase difference between the axial and tangential velocity fluctuations [26] may also play an important role.

Although for complex systems, experimental measurements are currently preferred, computational simulations, if sufficiently accurate and fast, would offer the benefits of allowing stability predictions prior to experimental realisation. Possibilities for incorporating flame information provided by high-fidelity CFD into low order combustor models are only recently beginning to be exploited.

The use of computational methods for capturing the flame response has been more limited. The linear FTF of several flames has been extracted using URANS (Unsteady Reynolds-Averaged Navier-Stokes) simulations, giving reasonable results [27, 28, 29]. Armitage et al. [7] used URANS to obtain the FDF for the bluff body stabilised premixed flame experimentally investigated by Balachandran et al. [5], capturing qualitatively the shape of the response, but with a significant offset in the amplitude of the heat release rate fluctuations. Ruan et al. [30] used URANS to study the same case, implementing a more advanced combustion model. No Large Eddy Simulation (LES) investigations for this case have yet been performed. 
The flames in gas turbine combustors are turbulent. LES, although computationally expensive, is capable of capturing the turbulent mixing and unsteady behaviour vital to improved flame models. LES is now widely used to study turbulent premixed combustion more generally, not just in the context of combustion instability [1, 31, 32].

The need to include acoustic waves when considering the flame response means that compressible CFD approaches are typically chosen for flame response investigations. These, however, are limited by the speed of sound appearing in the CFL time step limit, meaning that small time steps are required. For low-Mach number flames, this causes a significant increase in computational cost. Incompressible codes are thus advantageous. To use an incompressible LES code to determine the FDF, simulations will be performed for a small domain within the combustor. At the upstream boundary of the LES domain, the acoustic velocity perturbation will be transformed to an incompressible (i.e. hydrodynamic) fluctuation for the purpose of the flame response [33]. This is justified as the flame response is well known to be unaffected by compressibility effects $[23,16,34]$. The response of the unsteady heat release of the flame to this velocity perturbation can then be found for different forcing frequencies and amplitudes, allowing the FDF to be obtained using incompressible simulations. Febrer et al. [33] used such a hybrid approach for coupling acoustic wave effects with incompressible LES for the flame response. The well-documented ORACLES test case [35, 36] was used for validation, exhibiting combustion instability at $50 \mathrm{~Hz}$. An acoustic network model [3] for the whole geometry was coupled with incompressible LES at the combustor inlet plane, with the acoustic wave amplitude at this plane matched to a hydrodynamic inlet forcing in the LES. The flame response was obtained only at the known instability frequency, and the hybrid approach provided predictions in reasonable agreement with the experimentally measured limit cycle amplitude. This was encouraging for the usefulness of the coupled hybrid approach. An incompressible LES solver was also used to study an acoustically forced flame by Krediet et al. [37, 38] under swirl premixed conditions. Experimentally, the acoustic forcing was provided by speakers mounted in the upstream duct: this was imitated numerically by introducing velocity excitation at the inlet in the simulations. No flame shape dynamics were considered. The predictions were found to have good agreement with experimental data. Building on these ideas, the present study uses an incompressible LES solver to study an acoustically forced premixed flame, and obtains the full FDF under conditions for which a detailed experimental comparison can be performed.

The present numerical study considers the target case of the bluff-body stabilised flame investigated experimentally by Balachandran et al. [6,5]. This case has the advantage of (i) having an "acoustically short" flame within the combustor (unlike, for example, the long flame in the ORACLES combustor [35, 36]), which simplifies extraction of the unsteady heat release rate and future coupling with acoustic models (ii) having significant experimental data, including flame shape images, available for validation and (iii) having controlled acoustic boundary conditions to facilitate future comparison with limit cycle amplitudes (although these are only available for a partially premixed flame due to possible flame flash back under premixed conditions). The flame is acoustically forced with forcing velocity amplitude up to $64 \%$ of the mean flow. Thus far, only URANS simulations [7, 30] have been conducted and only at the two forcing frequencies of $40 \mathrm{~Hz}$ and $160 \mathrm{~Hz}$. The objectives of the paper are: (1) perform LES studies of the case for the first time and to compare these with experimental data and URANS results; (2) validate the numerical approach of LES for turbulent premixed flame simulation based on an open-source CFD software, Code_Saturne $[39,40]$; (3) calculate the full FDF of the flame response using LES, and to compare these to the experimental results.

The target case will be described in section 2, followed by the numerical details of the LES simulations in section 3. The cold flow results with and without acoustic forcing will be presented in section 4 . The reactive cases with and without acoustic forcing at frequencies of $40 \mathrm{~Hz}, 160 \mathrm{~Hz}, 240 \mathrm{~Hz}$ and $310 \mathrm{~Hz}$, will be given in section 5 . The full FDF is presented in section 6 and the discussion of the results for an elongated combustion chamber case is given in section 7. Conclusions are presented in the last section.

\section{Experimental test case}

The test case considered is an acoustically excited bluff-body stabilised turbulent flame within a combustor, as shown in Fig. 1. This is described in detail by Balachandran et al. [5, 6], and has featured in other studies of turbulent flames [41, 42, 43, 44]. A long tube emerges from a plenum chamber, within which is a conical bluff body with diameter $d=25 \mathrm{~mm}$. The air and gaseous ethylene are mixed far upstream to give a perfectly premixed mixture prior to the combustion. Combustion occurs in the "enclosure", an $80 \mathrm{~mm}$ long quartz cylinder of inner diameter 70mm which can avoid equivalence ratio $(\phi)$ variations due to the surrounding air entrainment. In the present numerical study, an equivalence ratio of $\phi=0.55$ is used, although other ratios were also investigated in the experiments. 


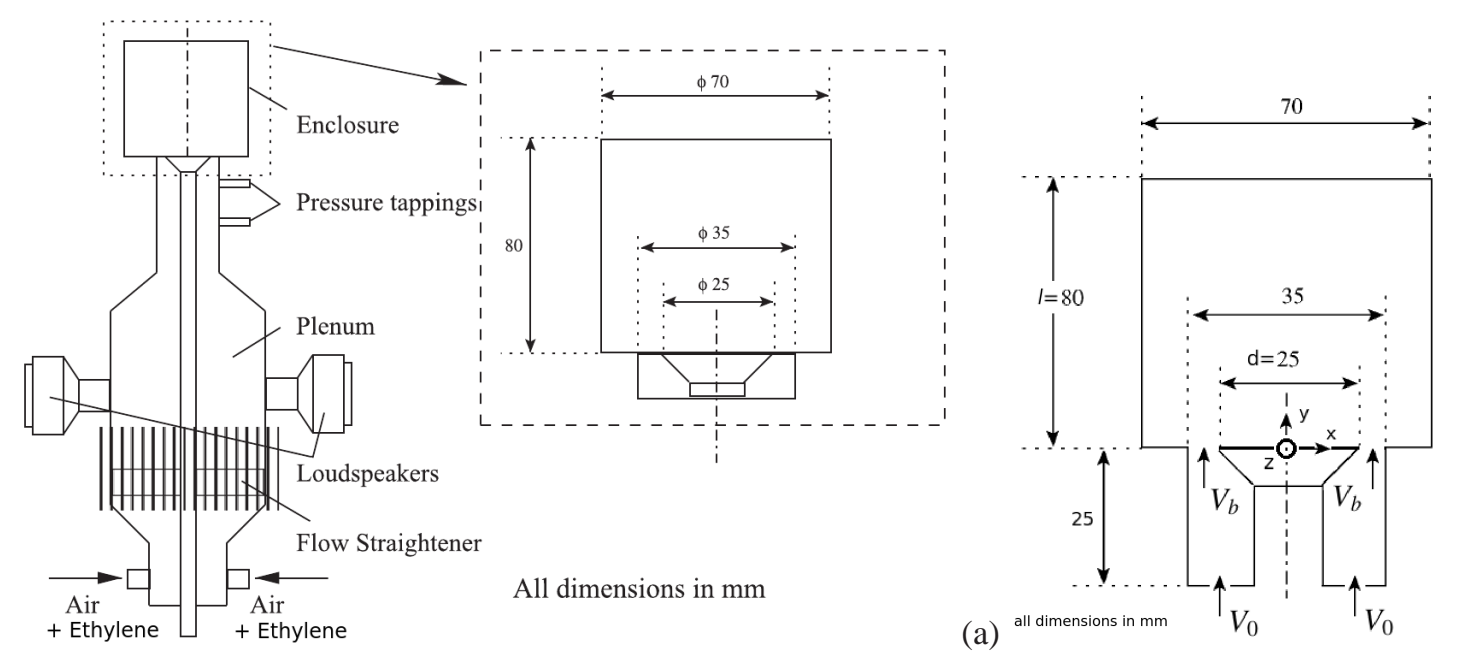

(b)

Figure 1: (a) Schematic of the experimental combustor test case [5]; (b) computational setup showing for the present LES studies a cut $(z=0)$ of the computational domain.

In the experiments, the acoustic forcing was generated by two loudspeakers, excited by a sinusoidal signal from waveform generator. This introduced velocity oscillations at the bluff body. Under premixed conditions, there are no equivalence ratio variations and the heat release rate fluctuations are dominated by velocity fluctuations upstream of the flame. Under the weakly non-linear assumption, for which forcing sinusoidally leads to a sinusoidal flame response at the same frequency (i.e. there is minimal generation of harmonics or subharmonics), the amplitude $(A)$ and the frequency $(f)$ of the forcing velocity fluctuation are then the two parameters upon which the heat release rate fluctuation depends. The normalised forcing amplitude and frequency were varied independently over a large range, with the forcing amplitude able to reach $64 \%$ of the mean velocity in the experiments. The experiments [5] suggested that the plenum chamber with the air supply line showed the features of a resonator with peak responses around 40, 160 and $320 \mathrm{~Hz}$. The heat release rate was measured with $\mathrm{OH}^{*}$ and $\mathrm{CH}^{*}$ chemiluminescence, and the phase-averaged FSD (Flame Surface Density) images obtained from the PLIF (Planar Laser-Induced Fluorescence) images were revolved around the burner central axis and were also used to obtain the normalised cyclic heat release fluctuation $[5,6]$.
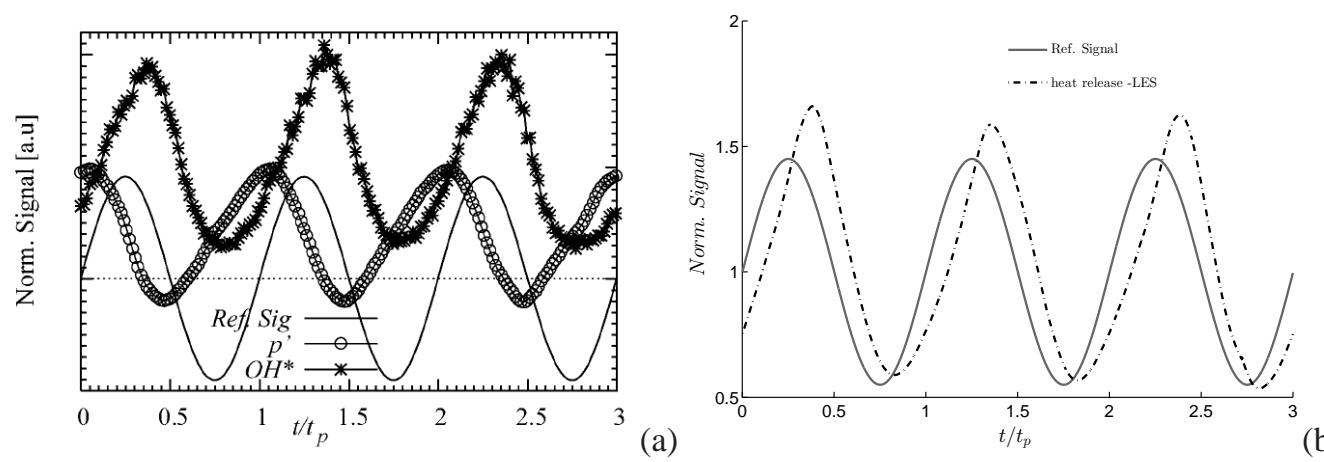

Figure 2: Time series of signals with sinusoidal forcing at $f=160 \mathrm{~Hz}$ and $A=0.45$ : (a) normalised reference velocity signal, acoustic pressure and $\mathrm{OH}^{*}$ chemiluminescence signals in the experiments of [5]; (b) normalised reference velocity signal and heat release rate signal from the present LES simulations.

To characterise the response of the flame, a velocity fluctuation upstream of the flame is used as the reference forcing signal in both the experiments and the present simulations. In the experiments, the reference velocity was taken at the combustor inlet (the position with the bulk velocity $V_{b}$ in Fig. 1(b)) determined from the acoustic pressure 
measurements using the two-microphone method. In the simulations, the reference velocity signal is taken at the computational inlet (the position with bulk velocity $V_{0}$ in Fig. 1(b)) but without the superimposed turbulent fluctuation. Figure 2 shows an example time series of reference forcing signals and resulting heat release rate signals in both experiments and the present simulation. The chemical source term $\tilde{\dot{\omega}}_{c}$ in Eq.(5) is integrated and recorded during the simulations to represent the heat release rate and an example signal is shown in Fig. 2(b). The signals are analysed spectrally using the Fourier Transform technique in order to determine the complex amplitude of the signals at the forcing frequency $f$. In the experiments, the values of $\mathrm{OH}^{* \prime}(\mathrm{f}) /\left\langle\mathrm{OH}^{*}\right\rangle$ and $\mathrm{CH}^{* \prime}(\mathrm{f}) /\left\langle\mathrm{CH}^{*}\right\rangle$ are used as estimates of $\mathrm{Q}^{\prime}(\mathrm{f}) /\langle\mathrm{Q}\rangle$, and the value of $\tilde{\dot{\omega}}_{c}^{\prime}(\mathrm{f}) /\left\langle\tilde{\dot{\omega}}_{c}\right\rangle$ is used to represent $\mathrm{Q}^{\prime}(\mathrm{f}) /\langle\mathrm{Q}\rangle$ in the present simulations. Based on the two quantities, heat release rate and reference velocity signal, the FDF defined in Eq.(2) can be determined.

The response of the cold flow field in the absence of a flame to velocity forcing will also be studied in the present paper. Experimental and LES data for this flow are available [45], and provide an additional means of validating the LES solver of Code_S aturne.

\section{Numerical method}

The present numerical simulations were large eddy simulations (LES) using the open source code Code_Saturne. The code has been used in several turbulent flow $[46,47,48]$ and turbulent combustion $[49,50,51]$ simulations. It is a parallel, general purpose, three dimensional low Mach number CFD code based on a finite volume method, with a collocated arrangement for all variables. In the cold flow case, the filtered Navier-Stokes equations are solved for the mass and momentum equations, while in the reactive case, a FSD combustion model is used and an additional equation describing the reaction progress variables solved.

The turbulence is modelled using the popular WALE (Wall-Adapting Local Eddy-viscosity) [52] LES subgrid scale model. The turbulent viscosity is given by:

$$
\mu_{t}=\bar{\rho}\left(C_{w} \Delta\right)^{2} \frac{\left(S_{i j}^{d} S_{i j}^{d}\right)^{3 / 2}}{\left(\tilde{S}_{i j} \tilde{S}_{i j}\right)^{5 / 2}+\left(S_{i j}^{d} S_{i j}^{d}\right)^{5 / 4}}
$$

where the model constant $C_{w}$ is equal to 0.5 [52], $\Delta$ is the cubic root of the cell volume, $\tilde{S}_{i j}$ is the strain rate of the resolved velocity. Term $S_{i j}^{d}$ is the traceless symmetric part of the square of the velocity gradient tensor $\tilde{g}_{i j}$, defined as:

$$
S_{i j}^{d}=\frac{1}{2}\left(\tilde{g}_{i j}^{2}+\tilde{g}_{j i}^{2}\right)-\frac{1}{3} \delta_{i j} \tilde{g}_{k k}^{2}
$$

with $\tilde{g}_{i j}=\partial \tilde{u}_{i} / \partial x_{j}$. Note that the symbol ${ }^{-}$denotes the spatial filtering used in LES and the symbol ${ }^{\sim}$ denotes densityweighted filtering defined as $\tilde{\phi}=\overline{\rho \phi} / \bar{\rho}$ for an arbitrary variable $\phi$.

Different models have been proposed for LES of premixed turbulent combustion, most of them being variants of the flamelet concept [31]. Recent developments in combustion models and relevant issues are reviewed in [53]. Previous work has confirmed that the flame relevant to the test case operates in the premixed combustion regime of the thin reaction zone [42]. It is thus appropriate to use a FSD combustion model for the simulations. In this method, all species, mass fractions and temperatures are linearly related and the combustion process can be described by a single scalar known as the progress variable, $\tilde{c}$, which has the range 0 to 1 [1]. $\tilde{c}=0$ represents the limit of fresh/unburned gasses while $\tilde{c}=1$ represents the limit of burned gasses. The governing equation of $\tilde{c}$ reads:

$$
\frac{\partial(\bar{\rho} \tilde{c})}{\partial t}+\frac{\partial\left(\bar{\rho} \tilde{u}_{i} \tilde{c}\right)}{\partial x_{i}}=\frac{\partial}{\partial x_{i}}\left[\left(\bar{\rho} D+\frac{\mu_{t}}{S c}\right) \frac{\partial \tilde{c}}{\partial x_{i}}\right]+\tilde{\dot{\omega}}_{c}
$$

where $D$ is the laminar diffusivity, $\mu_{t}$ the turbulent viscosity obtained from the WALE model (Eq. 3 ) and $S c$ the turbulent Schmidt number equal to 0.7 .

The complex turbulence and combustion interactions are represented by the modelling of the chemical source term $\tilde{\dot{\omega}}_{c}$ (Eq. (5)). In recent papers, some of the more popular algebraic FSD models are assessed in the context of LES based on DNS results or experimental data $[54,55]$. In the present study, the source term is modelled using:

$$
\tilde{\tilde{\omega}}_{c}=\bar{\rho}_{u} S_{l}^{0} \Xi \Sigma
$$


in which $\bar{\rho}_{u}$ is the density of unburned gas, $S_{l}^{0}$ the unstrained laminar flame speed, $\Xi$ the wrinkling factor and $\Sigma$ the FSD per unit volume. For the present ethylene and air premixed gas with an equivalence ratio of $\phi=0.55$, the unstrained laminar flame speed $S_{l}^{0}$ is determined by an empirical formula obtained from the experimental data [56].

The FSD $\Sigma$ corresponds to the resolved reaction rate estimated from filtered quantities such as $\tilde{c}$. An algebraic expression is used in the present work [57, 58]:

$$
\Sigma=4\left(\frac{6}{\pi}\right)^{1 / 2} \frac{\tilde{c}(1-\tilde{c})}{\left(\Delta^{2}+\delta_{l}^{2}\right)^{1 / 2}}
$$

where $\Delta$ is the mesh scale and $\delta_{l}$ is the laminar flame thickness which is introduced here to ensure the correct behaviour when $\Delta \rightarrow 0$ [59].

The wrinkling factor $\Xi$ measures the ratio of the total to the resolved flame surfaces in the filtering volume, or essentially the ratio of turbulent to laminar flame speed. The modelling of this wrinkling factor $\Xi$ can significantly affect the simulation predictions. Several models have previously been proposed [54, 55]. The one used in the present study gave the best fit to experimental data, and is based on Fureby's fractal approach [60]:

$$
\Xi=\left[\Gamma_{n}\left(\frac{u_{s g s}^{\prime}}{S_{l}^{0}}\right)\right]^{D_{f}-2}
$$

where $D_{f}$ corresponds to the fractal dimension with the value of 2.337 [58] and $\Gamma_{n}$ the efficiency function which accounts for the contribution from small vortices to wrinkle the flame front. The parameter $\Gamma_{n}$ is modelled by Angelberger et al. [61] as:

$$
\Gamma_{n}=0.75 \exp \left[-\frac{1.2}{\left(u_{s g s}^{\prime} / S_{l}^{0}\right)^{0.3}}\right]\left(\frac{\Delta}{\delta_{l}}\right)^{2 / 3}
$$

In the models (Eqs. (8) and (9)), an approximation of the subgrid-scale velocity fluctuation $u_{s g s}^{\prime}$ is required. As in the previous studies [55, 62], it is estimated using the relevant LES model:

$$
u_{s g s}^{\prime}=\frac{\mu_{t}}{\bar{\rho} C_{w} \Delta}
$$

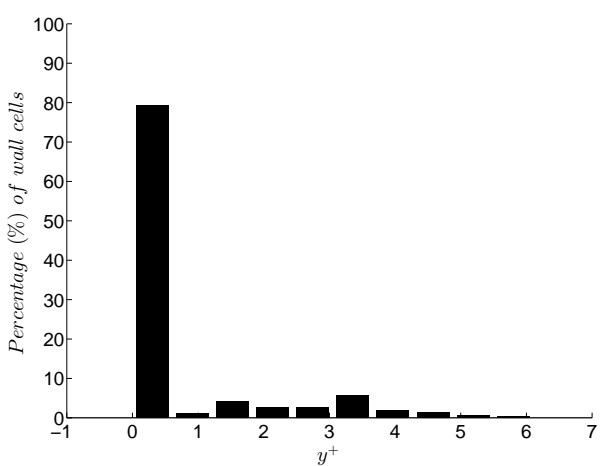

Figure 3: Statistics of the wall-normal distance, $y^{+}$, for all the wall cells.

The above FSD combustion model was implemented in Code_Saturne. All variables in the code are collocated at the cell centres and the SIMPLEC prediction/correction algorithm is used for pressure-velocity coupling. The convective terms are discretized using a second order central difference scheme and a second order upwind scheme was used for the scalar equation. The temporal advancement was approximated using a second order Crank-Nicolson implicit scheme. Version 3.0 of the code is used in this work - for more details refer to [39, 40]. 
The computational domain consists of the whole of the combustor (or "enclosure" in Fig. 1) and the upper $25 \mathrm{~mm}$ of the incoming circular duct. A $z$-cut of the computational domain is shown in Fig. 1(b) including the coordinate system used in the present simulations. Two different hexahedral meshes which contain about 1.2 million and 2.4 million cells, respectively, are used to check the mesh independence. The results which follow are from the finer mesh unless stated, in order to capture the small flow scales as accurately as possible. Meshes are clustered near the solid walls and the first node is located about $0.06 \mathrm{~mm}$ away from the walls. Figure 3 shows the statistics of $y^{+}$, i.e. the closest wall-normal distance of the nodes, from the simulation on the finer mesh. It can be seen that the majority are located within the viscous sublayer. Furthermore, the average of $y^{+}$is around 0.83 which is suitable for wall-resolved LES simulations. In the main reaction region (the first half of the combustor), the finer mesh has scales of around $(0.2 \mathrm{~mm}, 0.4 \mathrm{~mm}, 0.6 \mathrm{~mm})$ which lead to around $(5.5,11.0,16.5)$ wall units - these resolutions meet the requirements for typical wall-resolved LES simulations very well [63].

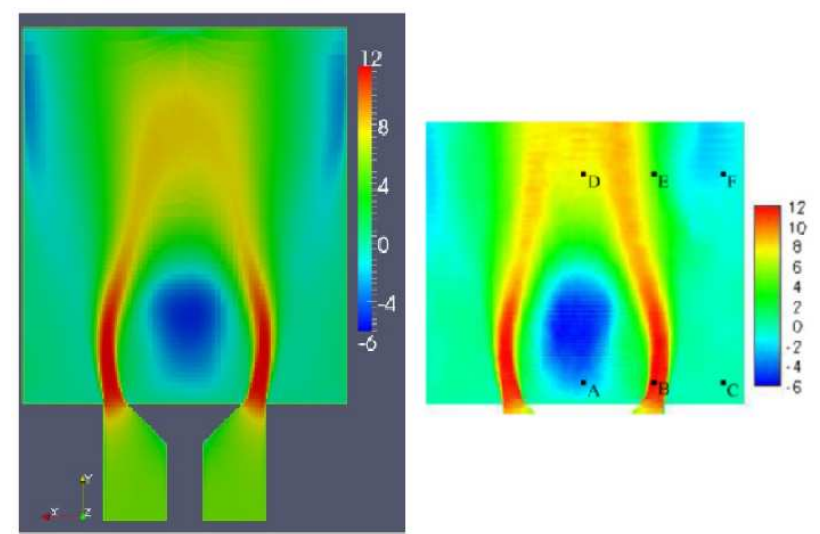

Figure 4: Comparisons of the time-averaged axial velocity $(\mathrm{m} / \mathrm{s}$ ) from the present LES (left) and previous LES (right) studies [45] for the unforced case. Points A-F are located at $(0,5),(15,50),(30,5),(0,50),(15,50)$ and $(30,50)$ (in $m m)$, respectively.

In the simulations, all boundaries other than the inlet and outlet are treated as solid walls, where non-slip wall conditions are applied. The walls are assumed to be adiabatic, meaning that heat loss is neglected. At the inlet, the velocity is imposed in the form of Eq.(11). In the experiments, the time-averaged bulk velocity entering the combustor was $V_{b}=9.9 \mathrm{~m} / \mathrm{s}$, giving a Reynolds number $R e=d V_{b} / v=17000$ [44]. Forcing was via pulsing of the flow upstream of the combustor by loudspeakers, such that a single frequency harmonic velocity is superimposed on the mean flow. Numerically, we represent this with a velocity profile in the annular passage at the simulation inlet of the form:

$$
V=V_{0}[1+A \sin (2 \pi f t)]+V_{t u r}^{\prime}
$$

where $A$ is the normalised velocity forcing amplitude and $f$ the forcing frequency. $A$ and $f$ are varied independently in the simulations in order to obtain the FDF. This form for the forcing has been used to simulate harmonic loudspeaker forcing of a flame in previous numerical studies [7, 33, 37, 38]. Note that $V_{b}$ is the mean velocity entering the combustion chamber and $V_{0}$ the mean velocity at the inlet of computational domain - these are related by mass balance such that $V_{0}=5.17 \mathrm{~m} / \mathrm{s}$ (see Fig. 1(b) for details). It was observed in the experiments [6, 44, 42] that considerable turbulent fluctuations were present in the incoming flow. The term $V_{t u r}^{\prime}$ in Eq. (11) is therefore modelled using a Synthetic Eddy Method (SEM) [64, 65], with amplitude 10\% of the mean velocity. The influence of this is addressed in Sec. 4. At the outlet, the default "outlet" boundary condition in Code_Saturne is applied: this consists of homogeneous Neumann conditions for velocity and scalars, and a Dirichlet condition for pressure (see refs. [39, 40] for more details).

\section{Results: cold flow}

The combustor flow field in the absence of a flame is firstly studied both in the unforced case and in the presence of acoustic forcing. Note that the experimental setup has also been used for studies of purely turbulent flow [45] and 
non-premixed combustion [66, 67]. Experimental data [67, 45] and reference LES results [66, 45] are available for the cold flow cases, providing an initial means of validating the present LES solver.

For the unforced case, the present LES predictions are compared with available experimental data [67] and two sets of previous LES studies [66, 45]. The time-averaged axial ( $y$-direction) velocity field is compared to that previously obtained from LES [45] in Fig. 4. Note that the two simulations employ different subgrid scale models (the present LES uses the WALE model while the previous LES uses dynamic Smagorinsky). The two LES studies predict similar main flow structures which can be distinguished clearly, including the annular jet, two shear layers and the central and outer recirculation zones. Agreement is good in the first half of the computational region, falling off at the top of the internal recirculation zone.

Further validation of the present LES solver is performed using the previous cold flow LES study of [66]. Fig. 5 compares the radial profiles of the mean and RMS axial velocities at three distances from the bluff-body, i.e. $y / d=$ $0.22,1.0,2.0$, respectively. The experimental data [67] are also included for comparison. A SEM free LES study is also performed to explore the effects of the incoming turbulence fluctuations. For both the mean and RMS axial velocities, the agreement of the present LES with experimental data and previous LES results is good when the SEM is applied, but weakened when it is not. This confirms that the SEM should be applied in the simulations. For the RMS velocities, the present LES underpredicts the experimental data, something that was also observed for the previous LES study.
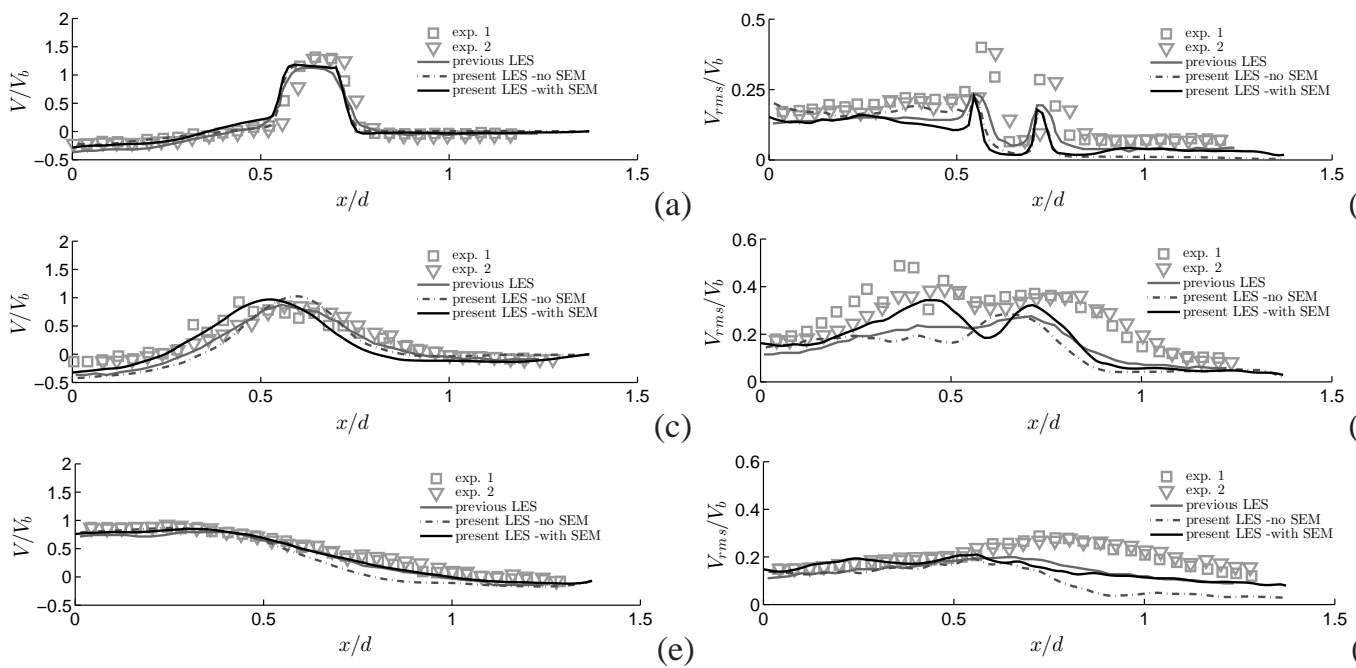

Figure 5: Comparisons of mean $\left(V / V_{b}\right)$ and RMS $\left(V_{r m s} / V_{b}\right)$ axial velocities by the present LES, previous LES and experimental studies for the unforced cold flow case at three locations: (a) $V / V_{b}$ at $y / d=0.22$, (b) $V_{r m s} / V_{b}$ at $y / d=0.22$, (c) $V / V_{b}$ at $y / d=1.0$, (d) $V_{r m s} / V_{b}$ at $y / d=1.0$, (e) $V / V_{b}$ at $y / d=2.0$ and (f) $V_{r m s} / V_{b}$ at $y / d=2.0$. The previous LES results come from ref. [66] and the experimental data from ref. [67].
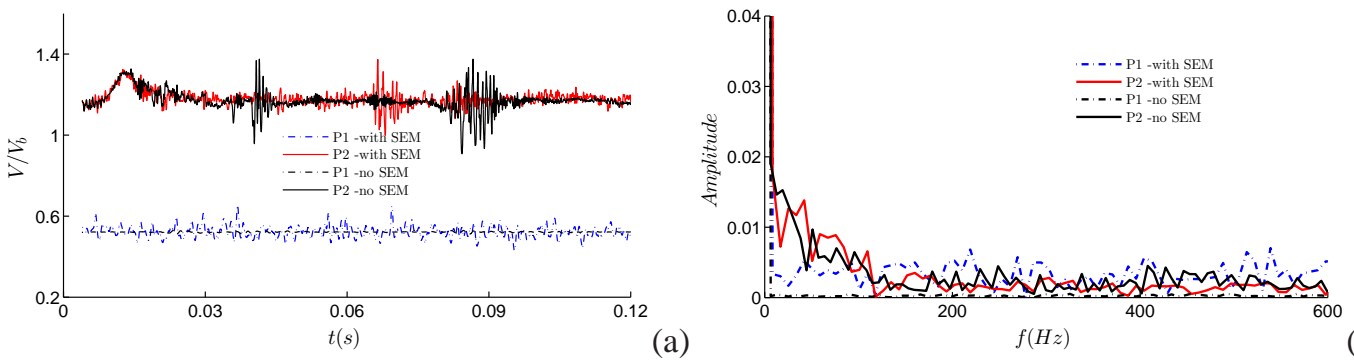

Figure 6: (a) examples of the axial velocity signals at two points for the unforced cold flow case from the present LES with/without SEM and (b) their Fourier Transform amplitudes. The two points are located at P1(10mm, -20mm, 0mm) and P2 (15mm, 5mm, 0mm).

By using a SEM, turbulent fluctuations are introduced at the inlet and it is possible that the frequency spectrum 
of the flow may be contaminated. In the simulations, the axial velocity signals at two points, P1(10mm, $-20 \mathrm{~mm}$, $0 \mathrm{~mm}$ ) located $5 \mathrm{~mm}$ downstream of the computational inlet and P2 $(15 \mathrm{~mm}, 5 \mathrm{~mm}, 0 \mathrm{~mm})$ located $5 \mathrm{~mm}$ downstream of the combustor inlet, are recorded. The signals and their Fourier Transform amplitudes are shown in Fig. 6. The SEM introduces some noise at P1, but the frequency range of interest to the present simulations, $(40 \mathrm{~Hz}, 400 \mathrm{~Hz})$, is largely unaffected. At P2, the SEM changes the evolution of the velocity signal considerably, but again the Fourier amplitudes are not significantly affected in the frequency range of interest, thus allowing the conclusion that noise introduced by the inlet SEM is not significant in the frequency range of interest.

It is also possible to compare one forced cold-flow case with both previous LES and experimental results. The forcing amplitude and frequency are $A=0.60$ and $f=160 \mathrm{~Hz}$ respectively (see Eq. (11)). The frequency is close to the resonance of the plenum in the experiments $[5,45]$. Coupled with the high forcing amplitude, this means that a flow field corresponding to a reactive case in which the flame response is well into the non-linear regime is being considered [6].
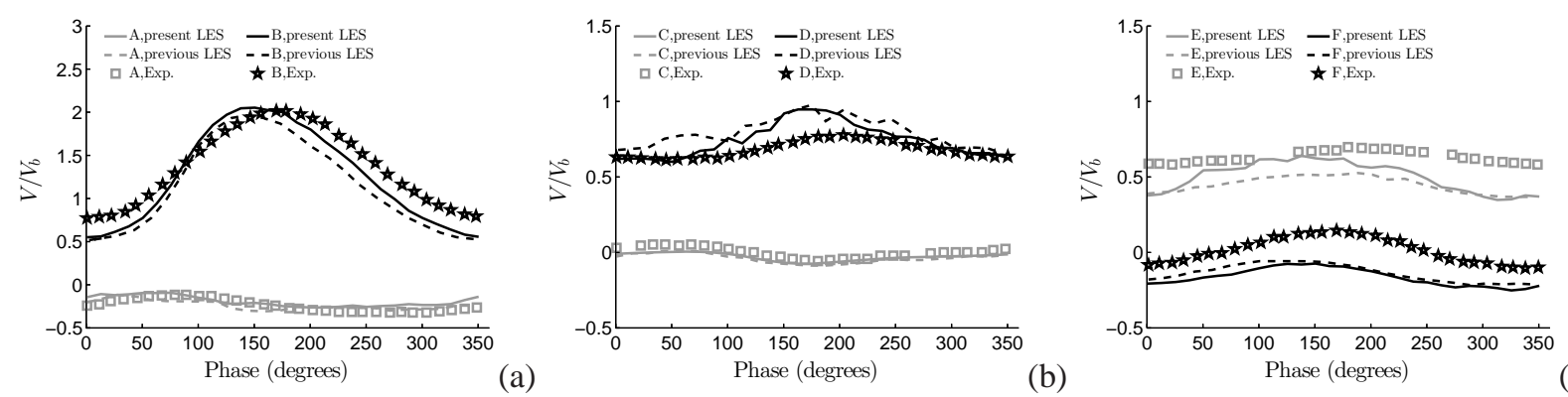

Figure 7: Comparisons of phase-averaged mean axial velocities by the present LES, previous LES and experimental studies [45] for the forced case. The first two points are given in (a), middle two points in (b) and last two points in (c). Cold flow case with acoustic forcing.
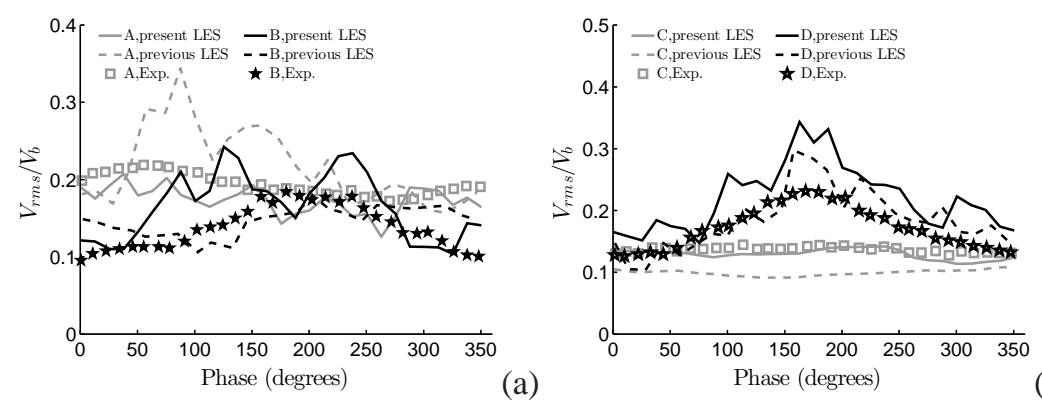

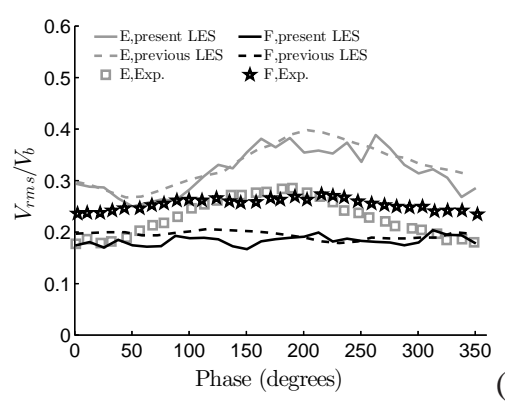

(c)

Figure 8: Comparisons of phase-averaged RMS axial velocities by the present LES, previous LES and experimental studies [45] for the forced case. The first two points are given in (a), middle two points in (b) and last two points in (c). Cold flow case with acoustic forcing.

Six points $(A$ to $F)$ are marked in different regions of the flow in Fig. 4, and are used to study the flow dynamics during excitation. The phase-averaged mean and RMS axial velocities are compared to previous LES [45] and experiments [45] in Figs. 7 and 8 respectively. For the mean velocities, the present LES predictions agree well with experimental data for all six points and over all phases - better than the previous LES results overall. The mean velocity responds as a harmonic oscillation, with a strong pulsation in the annular jet at point $\mathrm{B}$. The present LES results differ from the experiments more at the downstream three points, $D, E, F$. This may result from the possibility of reversed flow at the exit (see the recirculation region at the top region in Fig. 4 for reference) - reversed flow is not well resolved by the present nor previous LES studies. The RMS velocities at points $A$ and $C$ are well predicted by the present LES, but over-estimated at point $B$, suggesting that an improved turbulence model at inlet may be desirable. For the downstream three points, $D, E, F$, the present LES predictions generally agree well with the previous LES results, with both differing quite significantly from the experimental data. The reversed flow may also be affecting the RMS predictions. The phase of the RMS fluctuations closely follows that of the mean velocity. These results 
confirm that predictions from the present LES compare very well to the previous LES for this forced cold flow case. The comparison with experimental data is good for mean velocity and acceptable for the RMS fluctuations.

Overall, the results from the cold flow fields demonstrate that the present LES solver and mesh capture the flow field as accurately as the previous LES study, confirming that it is reasonable to consider reactive simulations using the present method.

\section{Results: reactive flow}

The unforced reactive case is firstly studied followed by forced cases at frequencies of $f=40,160,240$ and $310 \mathrm{~Hz}$. Flame snapshots and the non-linear flame response at the relevant forcing frequency are all compared to experimental results $[5,6]$ and, where available, previous URANS calculations [7, 30].
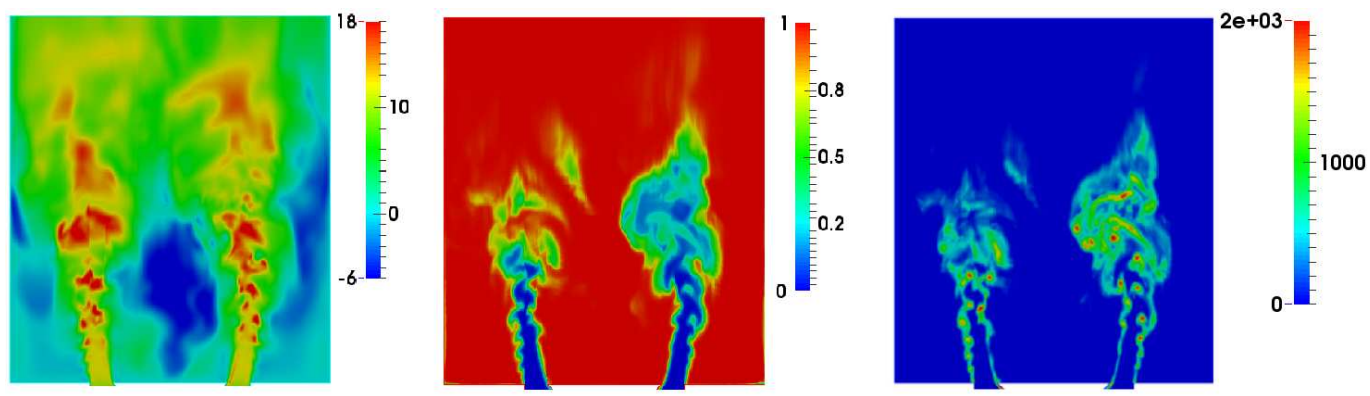

Figure 9: Snapshots of the unforced reactive flow field from the present LES, the axial velocity $V(\mathrm{~m} / \mathrm{s}$ ) (left), the progress variable $\tilde{c}$ (middle) and the chemical source term $\tilde{\dot{\omega}}_{c}$ (right).

\subsection{Unforced reactive case}

For the unforced simulations, the heat release rate strength per unit volume is described using the the chemical source term $\tilde{\dot{\omega}}_{c}$. Figure 10 shows contour plots comparing the time-averaged FSD image from experiments [6], the present LES and previous URANS simulations [7]. The snapshots of the axial velocity, the progress variable $\tilde{c}$ and the chemical source term $\tilde{\dot{\omega}}_{c}$ from the present LES are given in Fig. 9 for reference. They show that the flame is anchored at the shear layers from the inner bluff body and the outward backward-facing step. The present LES predicts that the flame extends about $2.0 \mathrm{~d}$ downstream of the bluff body while a much longer flame is given by previous RANS simulation. Compared with the experiments, the present LES gives a better prediction of the flame, although the length is slightly under-predicted. From Fig. 10, the agreement with experiments is less good at the top of the flame region, and suggests that the combustion is occurring slightly too quickly. The fractal dimension used in modelling the wrinkling factor $\Xi$ (Eq. (8)) was taken to have value 2.337 from a previous study [58] - this may be slightly too large for the present computational configuration $[68,58,69]$. Due to the simulation neglecting heat loss effects, it predicts a higher temperature in the outer recirculation region over the backward-facing step than in the experiments, thus the hotter gases push the flame away from the vertical wall in the simulation.

The complex unsteady vortex and flame interactions are evident in Figs. 10 and 9 from the LES results. Compared the velocity contours of the unforced cold flow case (Fig. 4) and unforced reactive flow case (Fig. 9), it is seen that the hot burned gasses right above the bluff body expand the fluid flow and push the shear layers towards the outer vertical walls. The shear layers are thus not connected in the reactive case as in the cold flow case. Furthermore, the central and outer recirculation regions are changed significantly by the heat release.

The adiabatic condition is used in the simulations, and thus the temperature is linearly linked with the progress variable $\tilde{c}$, i.e. $T=(1-\tilde{c}) T_{u n}+\tilde{c} T_{a d}$, where $T_{u n}$ is the unburned (fresh) gas temperature and $T_{a d}$ the adiabatic temperature (around $1713 \mathrm{~K}$ in the present case) of the burned gas. The plot of $\tilde{c}$ in Fig. 9 is thus proportional to that of the temperature. It shows that the temperature has the maximum value of $T_{a d}$ within the outer recirculation regions which makes a strong chemical reaction around the outer shear layer (see the middle figure in Fig. 10). However, in the experiments there is heat loss over the wall regions especially those of the backward-facing step. The gas in the 

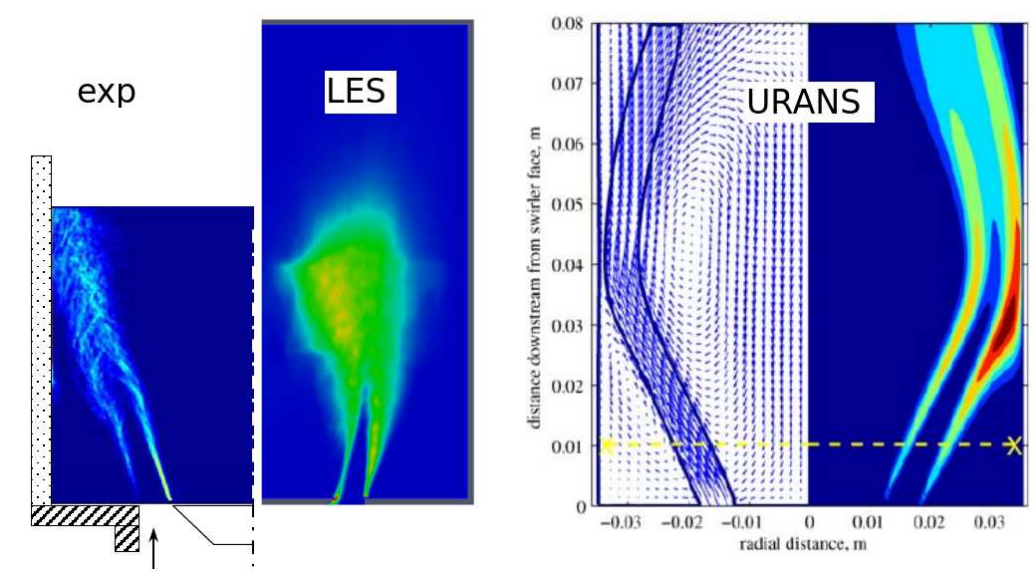

Figure 10: Time-averaged results of the unforced reactive flow field. FSD image from experiment [6] (exp), the chemical source term $\tilde{\tilde{\omega}}_{c}$ from the present simulation (LES) and the velocity vectors and heat release rate from previous URANS simulations [7].

outer recirculation region has a lower temperature in the experiment and thus the flame anchored by the outer shear layer has much lower chemical reaction than the one by the inner shear layer (see the left figure in Fig. 10). The stronger chemical reaction around the outer shear layer in the present LES study may lead to higher level of mean heat release, decreasing the effective magnitude of the FDF response in the forced cases [7].
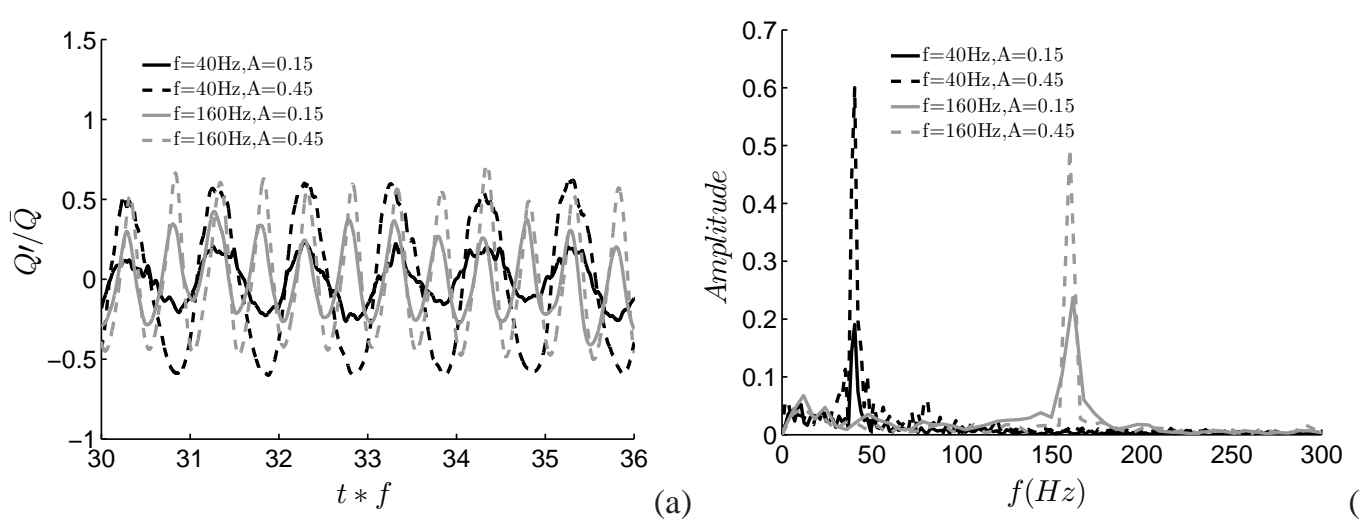

Figure 11: (a) examples of heat release rate signals at different acoustic forcing conditions from present LES. The time is non-dimensioned with the respective forcing frequency in (a), and (b) their amplitudes from Fourier Transform.

\subsection{Forced reactive cases}

The forced reactive cases are simulated by imposing velocity fluctuations on the mean velocity at the inlet, following the form shown in Eq. (11). This section will focus on the forced cases at the four frequencies of $f=40,160$, 240 and $310 \mathrm{~Hz}$, for which more detailed comparison to experiments is possible. Note that the full FDF calculated in Section 6 will include many more frequencies than the four considered here. A broad range of forcing amplitudes, ranging from 0.05 up to 0.64 is considered. At two of the frequencies, $f=40 \mathrm{~Hz}$ and $160 \mathrm{~Hz}$, comparison to the two URANS studies by Armitage et al. [7] (URANS-1R hereafter) and Ruan et al. [30] (URANS-2R hereafter) is also possible and is included.

The heat release rate fluctuation is an important quantity in the present simulations. In the framework of FSD combustion model used here, it is calculated from the integration of the chemical source term $\tilde{\omega}_{c}$ (Eq. (5)):

$$
Q=H_{f} \int \tilde{\omega}_{c} d V=H_{f} \int \bar{\rho}_{u} S_{l}^{0} \Xi \Sigma d V
$$



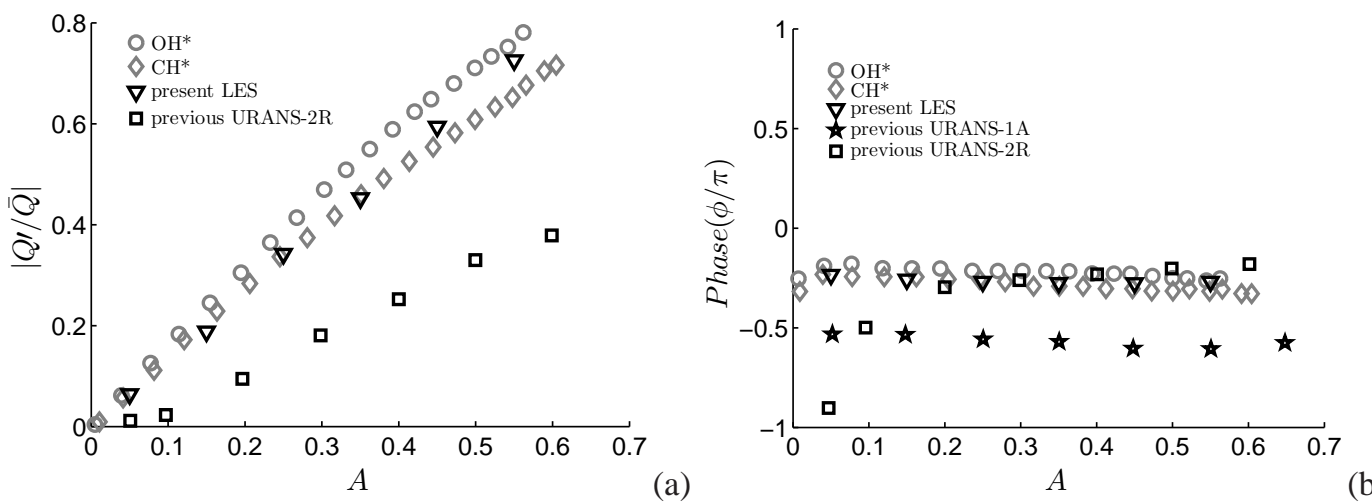

Figure 12: (a) Dependence of the amplitude of the heat release rate response with velocity fluctuation amplitude $A$; (b) the dependence of the phase of the heat release rate response $\varphi$ (Eq. (2)), at forcing frequency $f=40 \mathrm{~Hz}$. Experimental data are from [5], URANS-1A refers to results from [7] and URANS-2R from [30].

in which $H_{f}$ is the heat of formation. It should be noted that in the experiments, the integration area upon which the total heat release rate is based is that included in the observation window (of height $55 \mathrm{~mm}$ ) as opposed to the full combustor (height $80 \mathrm{~mm}$ ) [6]. Ruan et al. [30] studied the effect of the integration area on the fluctuating heat release rate in simulations using the URANS results, and found significant differences according to whether part (as in the experiments) or total combustor area was used. However, for the present LES, this difference is small (generally less than $4 \%$ ). This is because the predicted flame length is much shorter than for the URANS calculations and thus is mainly located within the observation area, as in the experiments. In the following sections, the heat release rate given by the present LES is the one from the total computation area. This is because the flame can be viewed as compact when incorporated in a low order acoustic solver and the total heat release is more relevant for further thermoacoustic studies.

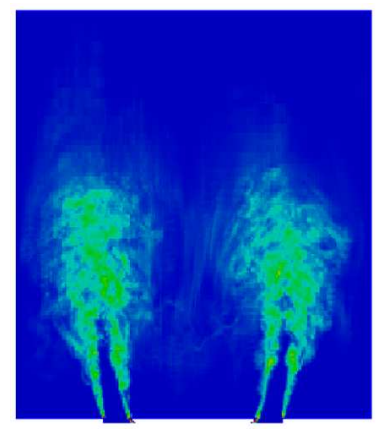

$\mathrm{f}=40 \mathrm{~Hz} \quad \mathrm{~A}=0.62$ $60^{\circ}$

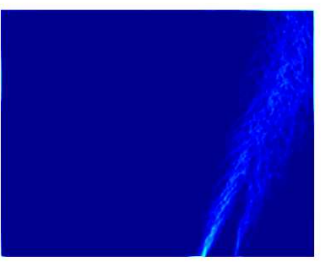

(a)

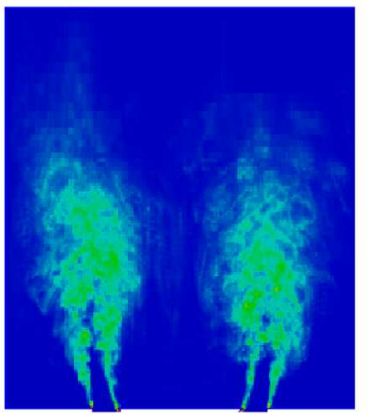

$f=40 \mathrm{~Hz} \quad A=0.62$ $120^{\circ}$

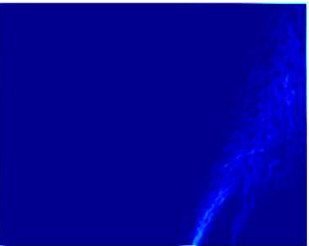

(b)

Figure 13: Comparisons of the mean chemical reaction rate from present LES (left) and phase-averaged FSD image from experiment [6] (right) at phases of $60^{\circ}$ and $120^{\circ}$ with strong acoustic forcing: $f=40 \mathrm{~Hz}$ and $A=0.62$.

The velocity at the combustor inlet contains a harmonic fluctuating component. Consequently, the forced combustion produces fluctuating heat release rate which is sampled during the simulation. The simulation results given in the following sections are based on at least 14 forcing cycles after the transients have died away: phase averaging is thus performed for at least 14 forcing cycles. The time series are transformed to the frequency domain using a Fourier Transform technique. The gain and phase of the FDF are obtained according to Eq. (2). Figure 11(a) shows signals of the instantaneous heat release rate under different forcing conditions by the present LES. The heat release rate responds differently to different forcing amplitudes and frequencies: it is predominantly harmonic at the forcing frequency (validating the assumption of weak non-linearity) with some cycle-to-cycle variation. The spectra of the signals shown in Fig. 11(b) confirms that the dominant response of the heat release rate remains at the forcing 
frequency.
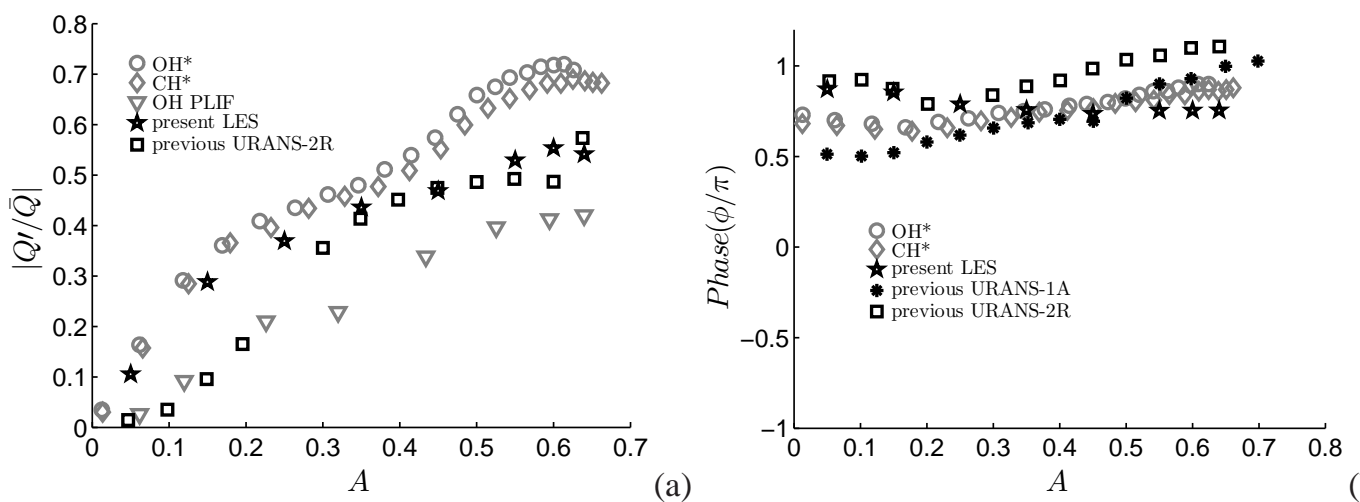

Figure 14: (a) Dependence of the amplitude of the heat release rate response with velocity fluctuation amplitude $A$; (b) the dependence of the phase of the heat release rate response $\varphi$ (Eq. (2)), at forcing frequency $f=160 \mathrm{~Hz}$. Experimental data are from [5], URANS-1A refers to results from [7] and URANS-2R from [30].

For a forcing frequency of $f=40 \mathrm{~Hz}$, simulations are performed for several velocity forcing amplitudes, $A$. The normalised amplitude of the heat release rate fluctuation as a function of forcing amplitude is shown in Figure 12, with comparisons to experiments and previous URANS studies included. It should be noted that the amplitude response of heat release in the previous URANS-1A study is normalized by its maximum absolute value, which is not reported, and so only the phase results are included [7]. Also note that the heat release rate in study URANS-2R [30] uses a truncated spatial integration window - this also applies to later results. The results show that the amplitude response is approximately linear up to $A=0.6$, with good agreement between the present LES predictions and experimental results from $\mathrm{OH}^{*}$ and $\mathrm{CH}^{*}$ chemiluminescence. The previous URANS-2R study produces the correct trend, but the amplitude of heat release fluctuation is much smaller. For the phase shown in Fig. 12(b), the experiment exhibits a nearly constant value of around $-0.25 \pi$ with increasing the forcing amplitude $A$. The present LES predicts this quite well, with obvious improvements over the URANS results. The FSD images in the experiment show that there is no shear layer rollup at this frequency [6]. Figure 13 shows the mean chemical reaction rate from the present LES and the FSD images from experiments at two phase angles of $60^{\circ}$ and $120^{\circ}$, with a high forcing amplitude of $A=0.62$. It can be seen that there is no obvious flame surface rollup. The LES predictions show that no big differences exist between the two phases, while the experimental results demonstrate a synchronized bulk oscillation of the flame.

The URANS-2R study underestimates heat release amplitude compared to the experiment at a forcing frequency of $f=40 \mathrm{~Hz}$, which may be due to the fact that combustion process is under-estimated in URANS-2R study. The present combustion model for LES will tend to over-estimate the heat release amplitude due to combustion being slightly too fast. However, the LES predictions agree well with experiment, implying that the heat loss in the experiments increases the heat release amplitude at this forcing frequency. The heat loss effects on the flame transfer function have been studied previously $[70,71,72]$ in which heat loss tends to increase the heat release amplitude at low forcing frequencies and decrease it at high forcing frequencies. For the present case forcing at low frequency of $f=40 \mathrm{~Hz}$, the observation from present LES study is consistent with previous studies [70, 71, 72].

For a forcing frequency of $f=160 \mathrm{~Hz}$, the flame response for different forcing amplitudes is shown in Figs. 14, 15 and 16. The response amplitude in Figure 14 shows that this time the response is non-linear, with the present LES producing much better agreement with the experiments than the previous URANS. The trend is captured very well, including the linear region up to around $A=0.15$, a transition region up to around $A=0.45$ followed by a saturation region with saturation occurring at around $A=0.6$. The previous URANS studies [7, 30] both fail to predict this saturation point. The amplitude of the heat release is under predicted slightly compared with the $\mathrm{OH}^{*}$ and $\mathrm{CH}^{*}$ chemiluminescence results from experiment, while its value is larger than the experimental data extracted from PLIF images. For forcing at $f=160 \mathrm{~Hz}$, the present LES neglects the heat loss and has a combustion model which is slightly too fast. Similar to the case forcing at a forcing frequency of $f=40 \mathrm{~Hz}$, it appears that the heat loss effect still dominates as the heat release amplitude is underestimated.

The phase response with forcing amplitude Figure 14(b) shows that all of the numerical methods produce reason- 

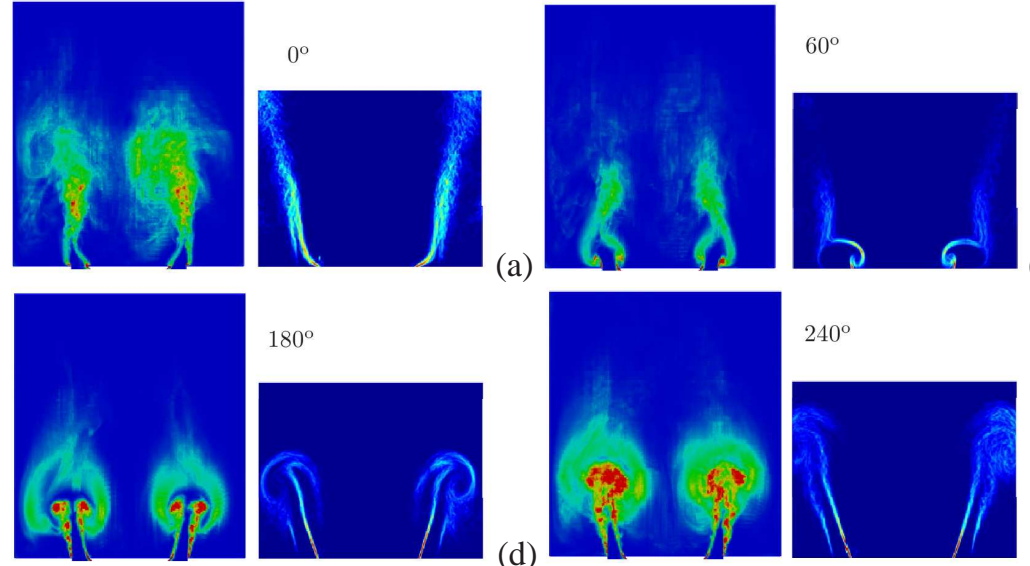

$240^{\circ}$

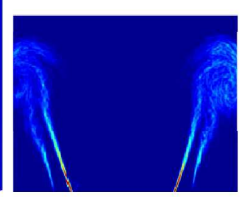

(b)

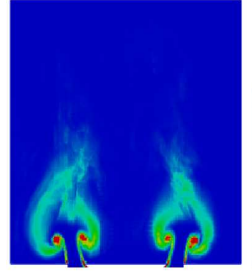

$120^{\circ}$

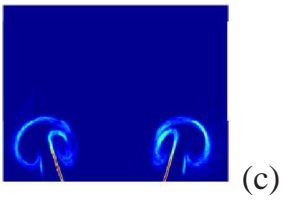

(e)

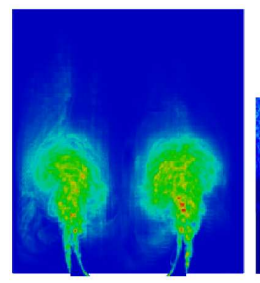

$300^{\circ}$

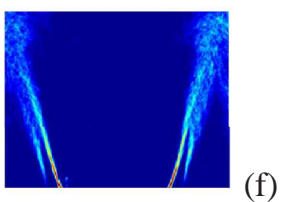

Figure 15: Comparisons of the mean chemical reaction rate from present LES (left) and phase-averaged FSD image from experiment [5] (right) at different phase angle with strong acoustic forcing: $f=160 \mathrm{~Hz}$ and $A=0.64$.

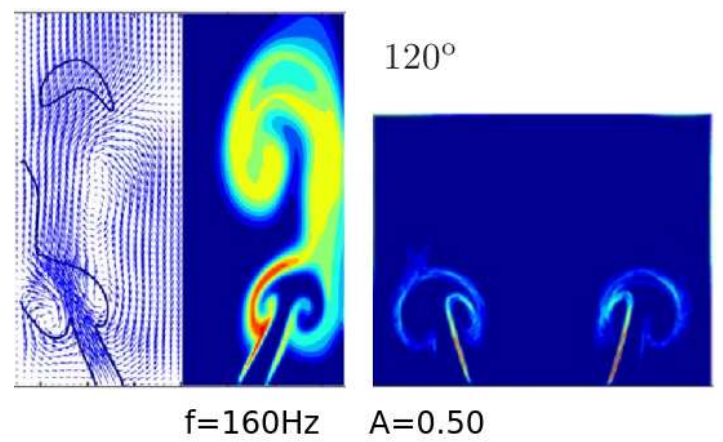

Figure 16: Phase-averaged FSD image at $120^{\circ}$ from the previous URANS-1A [7] (left) and experiment [6] (right) with strong acoustic forcing: $f=160 \mathrm{~Hz}$ and $A=0.50$. 
able results compared with experiment.
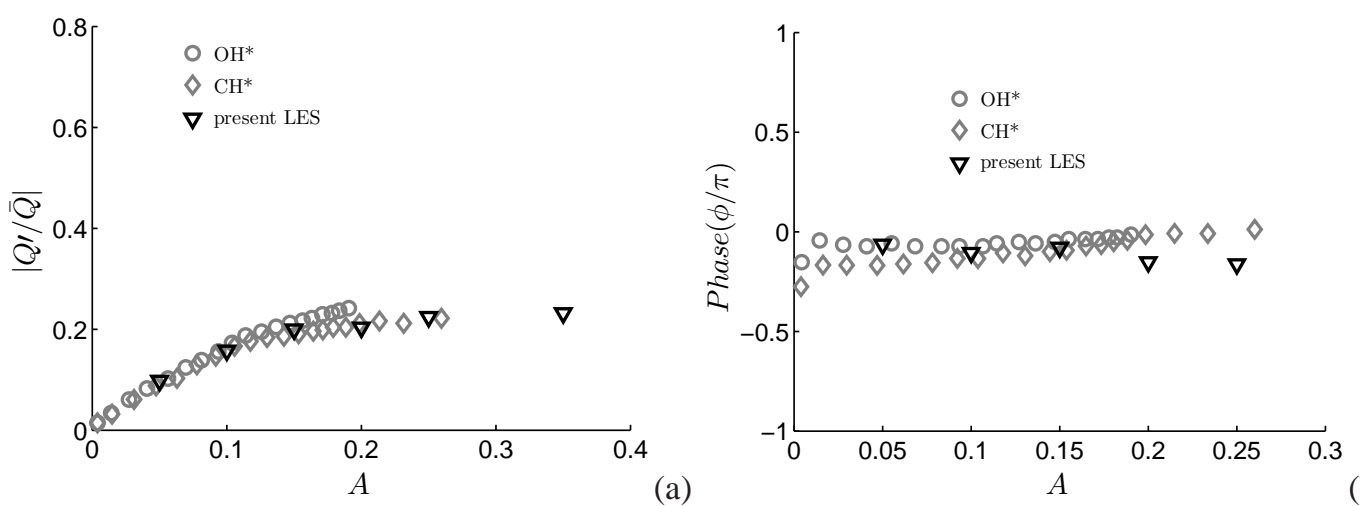

(b)

Figure 17: (a) Dependence of the amplitude of the heat release rate response with velocity fluctuation amplitude $A$; (b) the dependence of the phase of the heat release rate response $\varphi$ (Eq. (2)), at forcing frequency $f=240 \mathrm{~Hz}$. Experimental data are from [5].

Figure 15 visually shows the flame dynamics at a forcing frequency of $f=160 \mathrm{~Hz}$ and a forcing amplitude of $A=0.64$, corresponding to the saturation region (see Fig. 14). The present LES predictions are compared with experiments at every $60^{\circ}$ phase angle. The snapshots show that the flame is distorted dramatically during the forcing process. The inner and outer shear layers rollup and form a counter-rotating vortex pair. A mushroom-shaped contour evolution is evident during the process. At the first two phase angles of $0^{\circ}$ and $60^{\circ}$, the mushroom-shaped vortex starts to form at the base of the flame. At the same time, the flame above the vortex region starts to decrease, which suggests possible flame surface destructions in these regions. Following at the two phase angles of $120^{\circ}$ and $180^{\circ}$, the vortex continues to rollup and is convected downstream along the flame. The previous flame destruction regions nearly disappear. The inner and outer shear layers at the base of the flame seem to return to their unforced status. At the $240^{\circ}$ and $300^{\circ}$ phase angles, the vortex pairs move further downstream, start to collapse and then merge together. The inner and outer shear layers at the base move closer with the merging of the above vortex. Note that the inlet velocity and the heat release signals have a phase change of around $0.85 \pi$ (see Fig. 14(b)). The images at the phase angles of $60^{\circ}$ to $120^{\circ}$ show a small heat release rate while a large heat release rate appears at angles of $240^{\circ}$ to $300^{\circ}$. This goes some way to demonstrating how the vortex rollup distorts the flame area and results in a nonlinear response of the heat release rate. The effect of neglecting heat loss at the walls on the flame dynamics is also evident. The experiments show that the outer part of the vortex, located close to the walls, is much weaker than the central part, while the LES predictions have nearly the same strength; this is consistent with previous observations [73, 71]. Due to heat loss, the flame in experiments has a tendency to attach to the vertical walls during its evolution; the LES simulations are unable to capture this. Combustion modelling also has an impact on the flame dynamics: at phase angles of $0^{\circ}$ and $300^{\circ}$, the LES predicts a shorter flame than the experiment, consistent with the observations in the unforced reactive case. The flame dynamics as predicted by URANS [7] at forcing amplitude $A=0.50$ are shown in Fig. 16 at a phase angle of $120^{\circ}$; the flame appears rather long and distorted above the mushroom-shaped vortex compared to the experimental results.

To summarise, at a forcing frequency of $160 \mathrm{~Hz}$, the present LES method captures the non-linear response of the flame with good accuracy and also captures the flame dynamics correctly. The LES method shows obvious advantages over URANS for the predicting the non-linear flame response.

The dependence of the flame response on forcing amplitude at a forcing frequency of $f=240 \mathrm{~Hz}$ is shown in Fig. 17. The magnitude of the response is lower than at the previous two frequencies, and good agreement is observed between the LES predictions and experimental data. Heat release rate saturation again occurs, albeit at a lower forcing amplitude than at $160 \mathrm{~Hz}$. This may due to the fact that the forcing amplitude required to excite the shear layer rollup has strong frequency dependence, and the required amplitude decreases with increasing frequency [5, 74].

The evolution of the flame dynamics at $f=240 \mathrm{~Hz}$ at a forcing amplitude of $A=0.25$, at which point saturation has been reached, are shown in Fig. 18. The qualitative agreement with experiments is reasonable. A similar mechanism to that at $160 \mathrm{~Hz}$ is evident, although the vortex rollup is much weaker and expands the flow between the two shear 

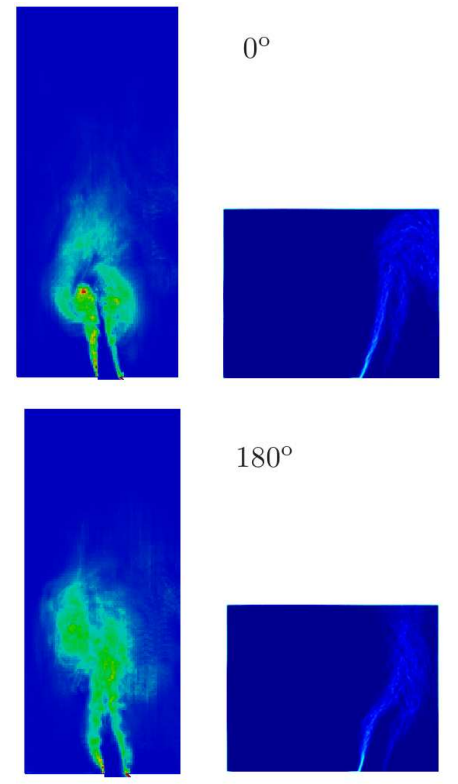

(a)
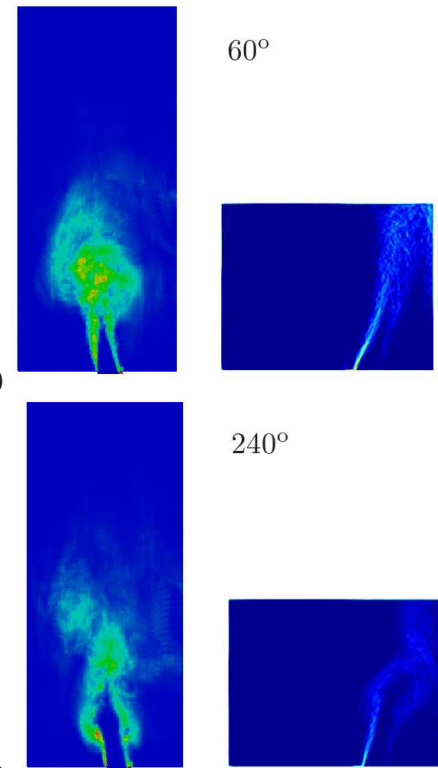

(d)

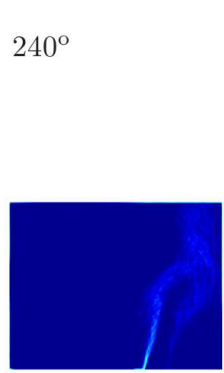

(b)
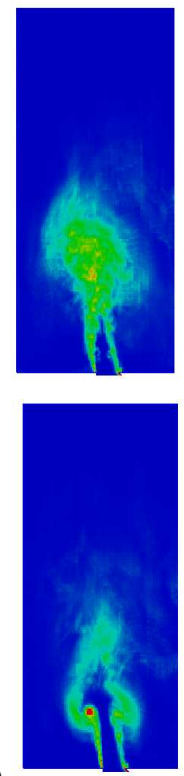

(e)

(f)

Figure 18: Comparisons of the mean chemical reaction rate from present LES (left) and phase-averaged FSD image from experiment [6] (right) at different phase angle with acoustic forcing: $f=240 \mathrm{~Hz}$ and $A=0.25$.

layers at $240 \mathrm{~Hz}$. The wrinkled flame front can be observed by the pulse of velocity perturbation. The results also confirm that heat loss makes the flame tend to attach to the cool wall: the present LES does not capture this behaviour well due to the lack of heat loss modelling. Similar to the observations at a forcing frequency of $f=40 \mathrm{~Hz}$, neglecting the heat loss and using a combustion model which is slightly too fast appear to balance in terms of the heat release rate prediction, as the results agree very well with the experiments.
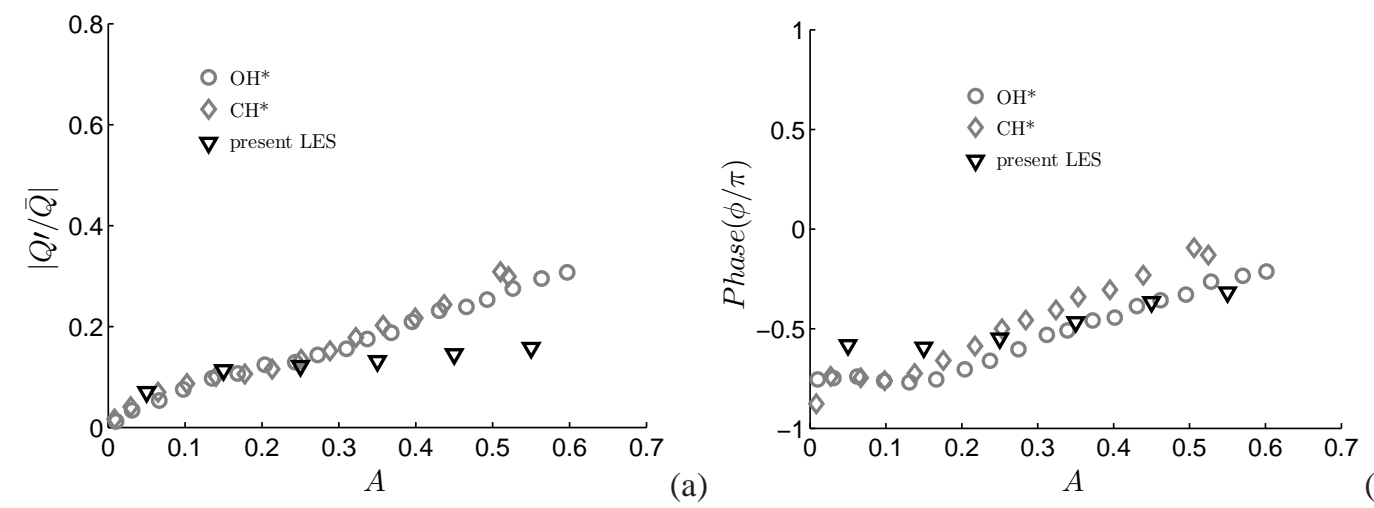

Figure 19: (a) Dependence of the amplitude of the heat release rate response with velocity fluctuation amplitude $A$; (b) the dependence of the phase of the heat release rate response $\varphi$ (Eq. (2)), at forcing frequency $f=310 \mathrm{~Hz}$. Experimental data are from [5].

At a forcing frequency of $310 \mathrm{~Hz}$, the dependence of the heat release rate on forcing amplitude is shown in Fig. 19. The magnitude of the response is again small compared to that at lower frequencies, and saturation, if indeed present, is much weaker. The agreement between the LES predictions and the experimental data is generally reasonable, although the LES implies a more pronounced saturation while the experiments appear to remain approximately linear. The phase also shows an approximately linear dependence, the trend of which is captured by the LES. Figure 20 compares the flame shape at $0^{\circ}$ phase angle with a high forcing amplitude of $A=0.62$ with the experiments [6]. A new vortex ("vor-2" in Fig. 20) is seen to appear before the old vortex ("vor-1" in Fig. 20) moves downstream out of 


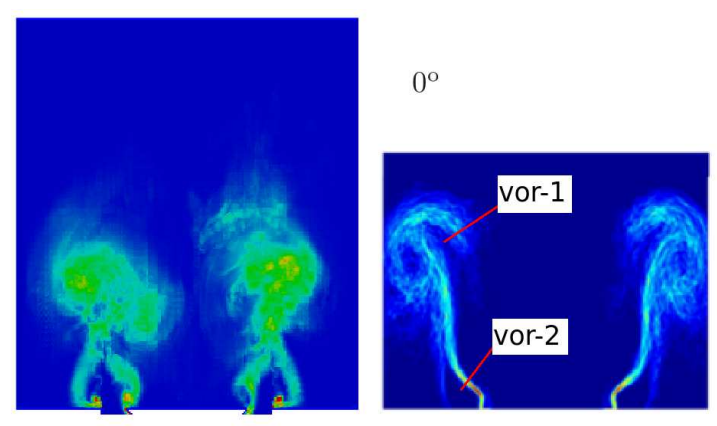

$f=310 \mathrm{~Hz} \quad A=0.62$

Figure 20: Chemical reaction rate image at $0^{\circ}$ from the present LES (left) and FSD image from experiment [6] (right) with strong acoustic forcing: $f=310 \mathrm{~Hz}$ and $A=0.62$. The two vortices are marked with "vor- 1 " and "vor-2", respectively.

the combustion zone, which means that there are two sets of vortex co-existing during one forcing period. The present LES captures this co-existence observed in the experiments [6], but the downstream vortex evolution is significantly affected by the cool walls and slower combustion - these are hard to distinguish from one another especially for the downstream vortex.

\section{Results of the full FDF}

The previous section showed that the LES method was able to capture the response of the unsteady heat release rate to acoustic forcing better than any numerical method to date, both in terms of the the main qualitative features of the flame dynamics, and the quantitative response. By performing many more simulations over frequencies ranging from $40 \mathrm{~Hz}$ to $380 \mathrm{~Hz}$, the full FDF can be simulated for this combustor.
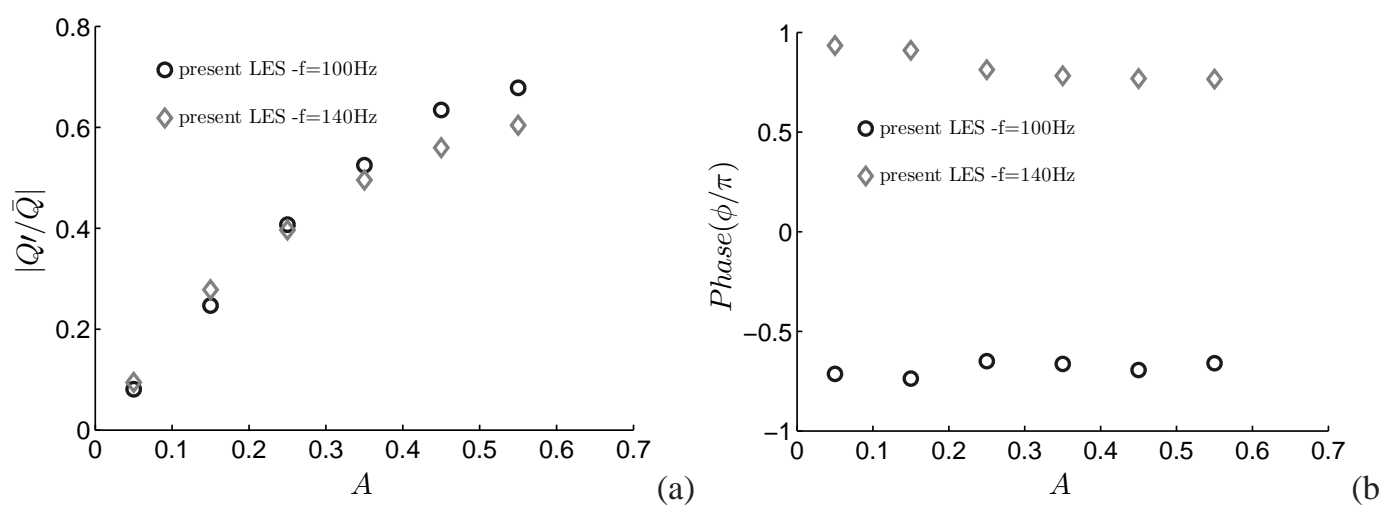

Figure 21: (a) Dependence of the amplitude of the heat release rate response with velocity fluctuation amplitude $A$; (b) the dependence of the phase of the heat release rate response $\varphi$ (Eq. (2)), at forcing frequency $f=100 \mathrm{~Hz}$ and $f=140 \mathrm{~Hz}$, from the present LES.

Further simulations at $100 \mathrm{~Hz}, 140 \mathrm{~Hz}$ and $380 \mathrm{~Hz}$ with forcing amplitudes ranging from 0.05 to 0.55 were performed. For forcing frequencies of $f=100 \mathrm{~Hz}$ and $f=140 \mathrm{~Hz}$, the dependence of the flame response on forcing amplitude is shown in Fig. 21. The amplitude of the heat release rate response at $f=100 \mathrm{~Hz}$ is linear for small forcing amplitudes $(A<0.25)$, gradually becoming nonlinear. At $f=140 \mathrm{~Hz}$, the linear region ends sooner and the heat release rate is smaller at high forcing amplitudes $(A>0.35)$, compared with forcing at $f=100 \mathrm{~Hz}$. The results clearly show the evolution of the heat release rate response from being fully linear at $f=40 \mathrm{~Hz}$ (see Fig. 12) to the combined linear response and saturation at $f=160 \mathrm{~Hz}$ (see Fig. 14). The phase results exhibit a jump from forcing at $f=100 \mathrm{~Hz}$ to $f=140 \mathrm{~Hz}$. The phase change is approximately forcing amplitude independent at $f=100 \mathrm{~Hz}$, while it gradually decreases with forcing amplitude $A$ at $f=140 \mathrm{~Hz}$. 

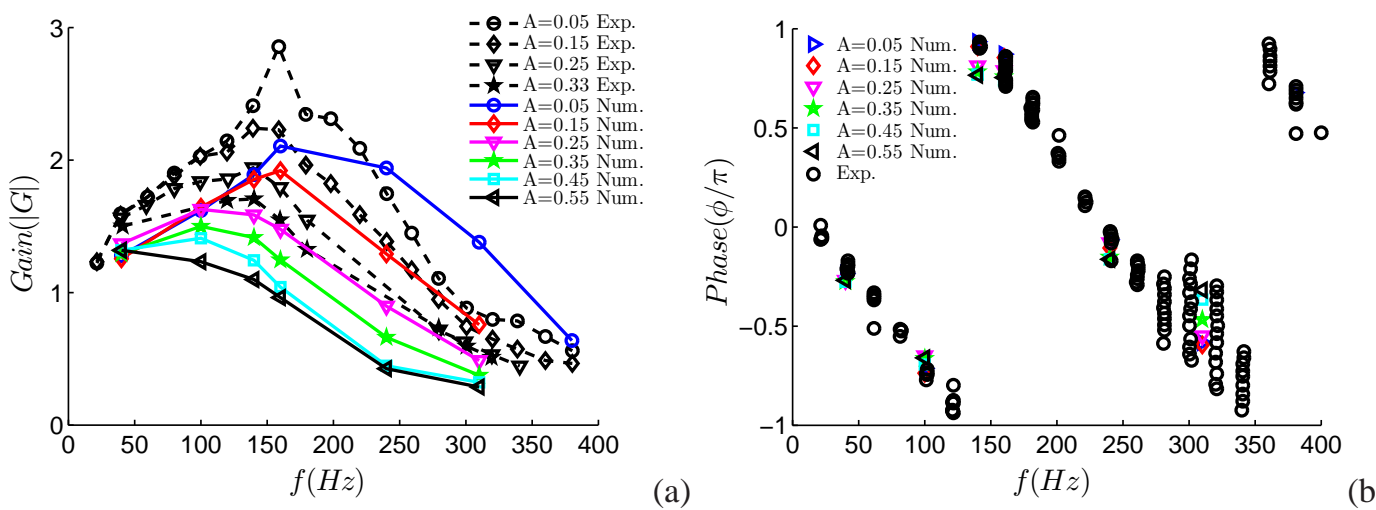

Figure 22: Comparison of the FDF results from present numerical method by LES (Num.) and experiment (Exp.): the gain (a) and the phase (b).

The full FDF defined in Eq. (2) is obtained, the gain and phase of which are shown in Fig. 22, including available experimental data. The simulation data is based on at least 14 forcing cycles after the simulation becomes fully developed. All the simulations use the same time step, except the lowest forcing frequency of $f=40 \mathrm{~Hz}$, where the time step is doubled. The total CPU time required to obtain all the simulation results is around 20400 hours. The LES predictions capture the correct trend of the gain, which rises and then falls off in frequency, except at the highest forcing amplitude where it only falls off with frequency, and decreases with forcing amplitude. The response magnitude is slightly under-estimated, most probably due to the heat loss effect and the combustion modelling issue discussed previously. This under-estimation is more obvious at small perturbations levels, indicating that a more realistic turbulence fluctuation model may be needed to improve accuracy in this regime. The nonlinearity of the gain is clearly evident - a linear response would not vary with forcing amplitude. The phase response implies a time delay which is estimated to have a value around $8 m s$ based on the phase slope Fig. 22(b): the LES results are in good agreement with the experimental data.

\section{Discussion of the long chamber case}

The simulations thus far address the flow configuration in which the length of the combustion chamber was $l=$ 80mm (see Fig. 1(b)). This configuration was chosen as detailed flame measurements for both the unforced and forced flow were available [6]. However, the ultimate aim of performing flame simulations is to facilitate predictions of the limit cycle amplitudes that occur in configurations that exhibit self-excitation. For the $l=80 \mathrm{~mm}$, chamber length (now termed the "short" chamber), the fundamental frequency is around $1000 \mathrm{~Hz}$, and self-excited oscillations do not occur. Increasing the length of the combustion chamber to $l=350 \mathrm{~mm}$ (termed the "long" chamber), resulted in combustion instability in the experiments (although only partially premixed flame experiments were performed due to the risk of flash back) [6]. The flame behaviour during limit cycle for this longer combustor length $(l=350 \mathrm{~mm})$ was observed to be very similar to that of the externally forced cases for the shorter combustor length $(l=80 \mathrm{~mm})$ at the same equivalence ratio and oscillation frequency. It is therefore interesting, in our fully premixed simulations, to see whether increasing the combustor length (and hence the length of our computational domain) significantly alters the FDF. If not, the FDF for the short combustor, which is computationally faster to calculate due to the smaller domain, can be used in place of the large domain FDF in low order thermoacoustic models, in order to obtain future limit cycle predictions.

Figure 23 shows the comparisons of the amplitude response of the heat release between the short chamber case $(l=80 \mathrm{~mm})$ and the long chamber case $(l=350 \mathrm{~mm})$ at the three frequencies of $40 \mathrm{~Hz}, 160 \mathrm{~Hz}$ and $310 \mathrm{~Hz}$. It can be seen that the heat release response for the long chamber is indeed very close to that for with short chamber at all the frequencies. The previous simulations (see Figs. 10, 13 and 15, etc.) showed that combustion occurs mainly in the first $3 / 4$ of the short chamber $(l=80 \mathrm{~mm})$, implying that the downstream flow field may not have large effects on the flame behaviour. These results suggest that the long chamber flame dynamics are very similar to those of the 

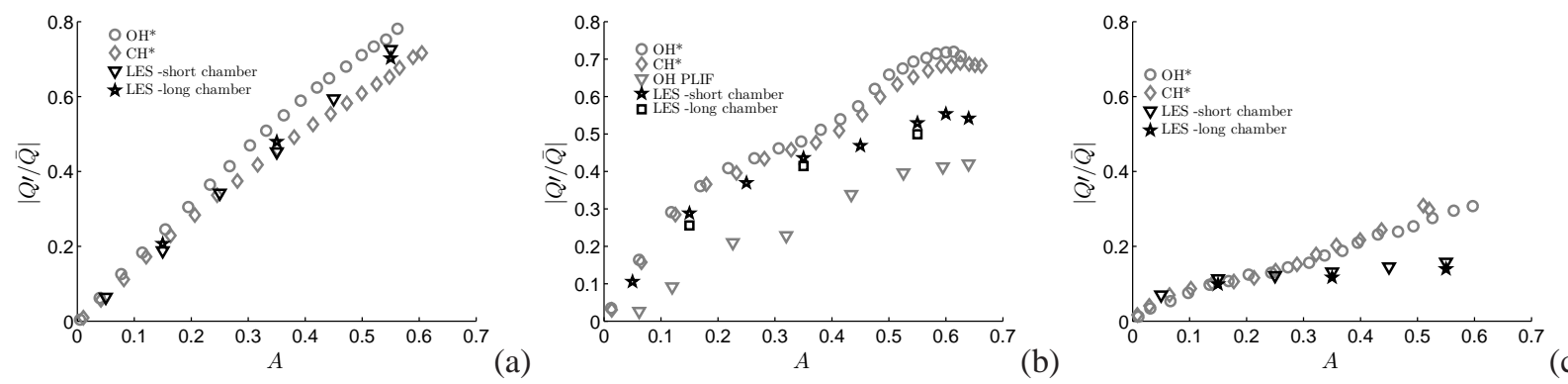

Figure 23: Comparison of the amplitude response of the heat release with forcing amplitude $A$ between the short chamber case and the long chamber case, at three forcing frequencies of $f=40 \mathrm{~Hz}$ (a), $160 \mathrm{~Hz}$ (b) and $310 \mathrm{~Hz}$ (c).

short chamber case. Thus the FDF for the short chamber could most probably be incorporated into thermoacoustic predictions for the long chamber with little penalty in accuracy.

\section{Conclusions}

Large Eddy Simulations, performed using an open source solver called Code_Saturne, were used to characterise the non-linear response of a turbulent premixed flame to acoustic forcing. A bluff-body stabilised, lean premixed flame, for which significant previous experimental data and some previous URANS data were available, was considered. This is the first work, to the authors' knowledge, which studies this particular flame using LES. The flame has the advantage of being acoustically short in length, and being enclosed in a combustor with simple acoustic boundary conditions, facilitating incorporation of the flame model into future low order combustor models for thermoacoustic predictions.

The LES method was validated using unforced/forced cold flow and unforced reacting data: both the flow and flame structures were captured well. Simulations were then performed which measured the response of the unsteady heat release rate to external inlet velocity forcing in order to extract the full Flame Describing Function (FDF). Four particular frequencies $40 \mathrm{~Hz}, 160 \mathrm{~Hz}, 240 \mathrm{~Hz}$ and $310 \mathrm{~Hz}$, were first studied in detail with normalised forcing amplitudes varying from 0.05 up to 0.64 . At the lowest forcing frequency, the flame responded linearly, while at intermediate frequencies non-linear behaviour is clearly observed. At high frequencies, the flame response falls off and the degree of non-linearity is unclear, but also less important. The LES results captured both the qualitative flame dynamics and the quantitative response of the heat release with reasonable accuracy - much better than has been obtained previously for this test case using numerical simulations. The main shortcomings of the simulations were felt to be the adiabatic wall boundary conditions and a combustion model that was slightly too fast: comparison with experimental flame dynamics suggested that wall heat loss and speed of combustion were likely to play important roles. Finally, it was found that increasing the combustor length had little affect on the flame response, suggesting that a flame model deduced from short-combustor (much faster) simulations could be used for long combustor combustion instability analysis.

This work confirms that open-source LES software, Code_Saturne, can be used to study the lean premixed combustion problems numerically, and good accuracy can be obtained.

\section{Acknowledgements}

This work is funded by the European Research Council via the ERC Starting Grant, ACOULOMODE (2013-18). Computation time using the CX1 HPC cluster at Imperial College is gratefully acknowledged, and we are grateful to Dr R. Balachandran of University College London and Prof. J. M. McGuirk of Loughborough University for several useful discussions. 


\section{References}

[1] T. Poinsot, D. Veynante, Theoretical and Numerical Combustion, R.T. Edwards, London, third edition, 2011.

[2] A. P. Dowling, S. R. Stow, Journal of Propulsion and Power 19 (2003) 751-763.

[3] S. R. Stow, A. P. Dowling, Journal of Engineering for Gas Turbines and Power 131 (2009) 031502.

[4] S. R. Stow, A. P. Dowling, in: Proceedings of ASME Turbo Expo 2004. GT2004-54245.

[5] R. Balachandran, B. Ayoola, C. Kaminski, A. Dowling, E. Mastorakos, Combustion and Flame 143 (2005) 37-55.

[6] R. Balachandran, Experimental investigation of the response of turbulent premixed flames to acoustic oscillations, Ph.d. thesis, University of Cambridge, Cambridge, UK, 2005.

[7] C. A. Armitage, R. Balachandran, E. Mastorakos, R. Cant, Combustion and Flame 146 (2006) $419-436$.

[8] L. Crocco, Journal of the American Rocket Society 21 (1951) 163-178.

[9] N. Noiray, D. Durox, T. Schuller, S. Candel, Journal of Fluid Mechanics 615 (2008) 139-167.

[10] S. Candel, Proceedings of the Combustion Institute 29 (2002) $1-28$.

[11] B. D. Bellows, M. K. Bobba, J. M. Seitzman, T. Lieuwen, Journal of Engineering for Gas Turbines and Power 129 (2007) $954-961$.

[12] A. Dowling, Journal of Fluid Mechanics 346 (1997) 271-290.

[13] D. Durox, T. Schuller, N. Noiray, S. Candel, Proceedings of the Combustion Institute 32 (2009) $1391-1398$.

[14] T. Lieuwen, Y. Neumeier, Proceedings of the Combustion Institute 29 (2002) 99-105.

[15] J. G. Lee, D. A. Santavicca, Journal of Propulsion and Power 19 (2003) 735-750.

[16] B. D. Bellows, Y. Neumeier, T. Lieuwen, Journal of Propulsion and Power 22 (2006) 1075-1084.

[17] A. L. Birbaud, D. Durox, S. Ducruix, S. Candel, Proceedings of the Combustion Institute 31 (2007) 1257-1265.

[18] S. Schimek, J. P. Moeck, C. O. Paschereit, Journal of Engineering for Gas Turbines and Power 133 (2011) 101502.

[19] D. Kim, K. Kim, S. Srinivasan, J. G. Lee, B. D. Quay, D. A. Santavicca, Journal of Engineering for Gas Turbines and Power 132 (2010) 021502 .

[20] B. Schuermans, F. Guethe, D. Pennell, D. Guyot, C. O. Paschereit, Journal of Engineering for Gas Turbines and Power 132 (2010) 111503.

[21] P. Palies, D. Durox, T. Schuller, S. Candel, Combustion and Flame 158 (2011) 1980-1991.

[22] C. F. Silva, F. Nicoud, T. Schuller, D. Durox, S. Candel, Combustion and Flame 160 (2013) 1743-1754.

[23] T. Lieuwen, Journal of Propulsion and Power 19 (2003) 765-781.

[24] P. Palies, T. Schuller, D. Durox, L. Y. M. Gicquel, S. Candel, Physics of Fluids 23 (2011) 037101.

[25] P. Palies, D. Durox, T. Schuller, S. Candel, Combustion Science and Technology 183 (2011) 704-717.

[26] T. Komarek, W. Polifke, in: ASME Turbo Expo 2009. GT2009-60100.

[27] C. A. Armitage, A. J. Riley, R. S. Cant, A. P. Dowling, S. R. Stow, in: ASME Turbo Expo 2004. GT2004-53820.

[28] M. Zhu, A. P. Dowling, K. N. C. Bray, Journal of Engineering for Gas Turbines and Power 127 (2005) 18-26.

[29] L. Tay-Wo-Chong, T. Komarek, R. Kaess, S. Föller, W. Polifke, in: ASME Turbo Expo 2010. GT2010-22769.

[30] S. Ruan, T. D. Dunstan, N. Swaminathan, R. Balachandran, in: 24th International Colloquium on the Dynamics of Explosions and Reactive Systems, 2013.

[31] H. Pitsch, Annual Review of Fluid Mechanics 38 (2006) 453-482.

[32] G. Wang, M. Boileau, D. Veynante, Combustion and Flame 158 (2011) 2199-2213.

[33] G. Febrer, Z. Yang, J. J. McGuirk, in: 47th AIAA/ASME/SAE/ASEE Joint Propulsion Conference \& Exhibit 2011. AIAA $2011-6127$.

[34] S. Ducruix, T. Schuller, D. Durox, S. Candel, Journal of Propulsion and Power 19 (2003) 722-734.

[35] M. Besson, P. Bruel, J. L. Champion, B. Deshaies, Journal of Thermophysics and Heat Transfer 14 (2000) 59-67.

[36] P. D. Nguyen, P. Bruel, S. Reichstadt, Flow, Turbulence and Combustion 82 (2009) 155-183.

[37] H. J. Krediet, C. H. Beck, W. Krebs, S. Schimek, C. O. Paschereit, J. B. W. Kok, Combustion Science and Technology 184 (2012) 888-900.

[38] H. J. Krediet, C. H. Beck, W. Krebs, J. B. W. Kok, Proceedings of the Combustion Institute 34 (2013) 1223 - 1230.

[39] F. Archambeau, N. Mechitoua, M. Sakiz, International Journal of Finite Volumes 1 (2004) 1-62.

[40] Y. Fournier, J. Bonelle, C. Moulinec, Z. Shang, A. G. Sunderland, J. C. Uribe, Computers \& Fluids 45 (2011) $103-108$.

[41] B. Ayoola, G. Hartung, C. A. Armitage, J. Hult, R. S. Cant, C. F. Kaminski, Experiments in Fluids 46 (2009) $27-41$.

[42] N. Swaminathan, G. Xu, A. P. Dowling, R. Balachandran, Journal of Fluids Mechanics 681 (2011) 80-115.

[43] J. Kariuki, J. R. Dawson, E. Mastorakos, Combustion and Flame 159 (2012) 2589-2607.

[44] G. Hartung, J. Hult, C. F. Kaminski, J. W. Rogerson, N. Swaminathan, Physics of Fluids 20 (2008) 035110.

[45] S. Ayache, J. R. Dawson, A. Triantafyllidis, R. Balachandran, E. Mastorakos, International Journal of Heat and Fluid Flow 31 (2010) $754-766$.

[46] I. Afgan, S. Benhamadouche, X. Han, P. Sagaut, D. Laurence, Journal of Fluid Mechanics 720 (2013) $457-485$.

[47] N. Lancial, F. Beaubert, S. Harmand, G. Rolland, International Journal of Heat and Fluid Flow 44 (2013) 336-347.

[48] A. Fadai-Ghotbi, C. Friess, R. Manceau, J. Borée, Physics of Fluids 22 (2010) 055104.

[49] H. Q. Dong, V. Robin, A. Mura, M. Champion, Flow, Turbulence and Combustion 90 (2013) 301-323.

[50] V. Robin, A. Mura, M. Champion, Combustion Science and Technology 184 (2012) 1718-1742.

[51] L. Gay, B. Sapa, F. Nmira, Fire Safety Journal 62, Part B (2013) 161-173.

[52] F. Nicoud, F. Ducros, Flow, Turbulence and Combustion 62 (1999) 183-200.

[53] L. Gicquel, G. Staffelbach, T. Poinsot, Progress in Energy and Combustion Science 38 (2012) 782 - 817.

[54] N. Chakraborty, M. Klein, Physics of Fluids 20 (2008) 085108.

[55] T. Ma, O. T. Stein, N. Chakraborty, A. M. Kempf, Combustion Theory and Modelling 17 (2013) 431-482.

[56] G. Abu-Orf, Laminar flamelet reaction rate modelling for spark ignition engines, Ph.d. thesis, University of Manchester Institute of Science and Technology, 1996.

[57] M. Boger, D. Veynante, H. Boughanem, A. Trouvé, Proceedings of the Combustion Institute 27 (1998) 917-925.

[58] D. Veynante, T. Schmitt, M. Boileau, V. Moureau, Analysis of dynamic models for turbulent premixed combustion, Technical Report, Center for Turbulence Research, Proceedings of the Summer Program 2012, 2012. 
[59] F. Charlette, C. Meneveau, D. Veynante, Combustion and Flame 131 (2002) 181-197.

[60] C. Fureby, Proceedings of the Combustion Institute 30 (2005) 593-601.

[61] C. Angelberger, D. Veynante, F. Egolfopoulos, T. Poinsot, Large eddy simulation of combustion instabilities in premixed flames, Technical Report, Center for Turbulence Research, Proceedings of the Summer Program 1998, 1998.

[62] F. C. Marincola, T. Ma, A. Kempf, Proceedings of the Combustion Institute 34 (2013) 1307-1315.

[63] P. Sagaut, Large eddy simulation for incompressible flows, An Introduction, Springer-Verlag, New York, third edition, 2006

[64] N. Jarrin, S. Benhamadouche, D. Laurence, R. Prosser, International Journal of Heat and Fluid Flow 27 (2006) $585-593$.

[65] N. Jarrin, R. Prosser, J. C. Uribe, S. Benhamadouche, D. Laurence, International Journal of Heat and Fluid Flow 30 (2009) $435-442$.

[66] A. Triantafyllidis, E. Mastorakos, R. Eggels, Combustion and Flame 156 (2009) 2328 - 2345.

[67] S. Ahmed, R. Balachandran, T. Marchione, E. Mastorakos, Combustion and Flame 151 (2007) 366 - 385.

[68] G. North, D. Santavicca, Combustion Science and Technology 72 (1990) 215 - 232

[69] O. Chatakonda, E. Hawkes, M. Brear, J. Chen, E. Knudsen, H. Pitsch, Modeling of the wrinkling of premixed turbulent flames in the thin reaction zones regime for large eddy simulation, Technical Report, Center for Turbulence Research, Proceedings of the Summer Program $2010,2010$.

[70] R. Kaess, W. Polifke, T. Poinsot, N. Noiray, D. Durox, T. Schuller, S. Candel, CFD-based mapping of the thermo-acoustic stability of a laminar premix burner, Technical Report, Center for Turbulence Research, Proceedings of the Summer Program 2008, 2008.

[71] L. Tay-Wo-Chong, W. Polifke, Journal of Engineering for Gas Turbines and Power 135 (2013) 021502.

[72] D. Mejia, L. Selle, R. Bazile, T. Poinsot, Proceedings of the Combustion Institute (2014). In press. http://dx.doi.org/10.1016/j.proci.2014.07.015.

[73] K. S. Kedia, H. M. Altay, A. F. Ghoniem, Proceedings of the Combustion Institute 33 (2011) 1113-1120.

[74] C. Külsheimer, H. Büchner, Combustion and Flame 131 (2002) 70-84. 